\title{
Universal Covers of Geometries of Far Away Type
}

\author{
ANTONIO PASINI \\ University of Siena, Department of Mathematics, Via del Capitano 15, 53100 Siena, Italy
}

pasini@unisi.it

Received March 7, 2002; Revised January 24, 2003; Accepted January 26, 2003

\begin{abstract}
The geometries studied in this paper are obtained from buildings of spherical type by removing all chambers at non-maximal distance from a given element or flag. I consider a number of special cases of the above construction chosen among those which most frequently appear in the literature, proving that the resulting geometry is always simply connected but for three cases of small rank defined over $G F(2)$ and $G F(4)$. I also compute the universal cover in those exceptional cases.
\end{abstract}

Keywords: buildings, universal covers, embeddings, binary codes

\section{Introduction}

Geometries obtained from buildings of spherical type by removing all elements at nonmaximal distance from a given element or a flag, are met in the context of many interesting characterizations and classifications. Many of them also appear in connection with embeddings of buildings of spherical type, as affine expansions of some of those embeddings (see [16]). As shown in [16], the hull of an embedding corresponds to the universal cover of the expansion of that embedding. In particular, an embedding is its own hull if and only if its expansion is simply connected.

In this paper I consider a number of special cases of the construction sketched above, proving that nearly all of the geometries obtained in those cases are simply connected. It is likely that the same conclusion holds for more families of 'far away' geometries, different from those studied here. In fact, I have only considered those families that either are related to some of the embeddings discussed in [16] or include examples that have been investigated by some authors in some contexts. Actually, my selection misses one family which however, according to the above criteria, deserved to be studied, namely the case of the subgeometry of a building of type $F_{4}$ far from a given point or symp. I have not considered it simply because I couldn't find the right way to treat it.

We follow [13] for basic notions and general results on geometries and Tits [18] for buildings. In particular, according to [13], we assume all geometries to be residually connected and firm, by definition.

We refer to chapters 8,11 and 12 of [13] for $m$-covers, $m$-quotients and $m$-simple connectedness, but we are only interested in 2 - and $(n-1)$-covers in this paper. We recall that the $(n-1)$-covers of a geometry of rank $n$ are called topological covers in [13], but many authors simply call them covers. We too do so in this paper. Accordingly, in the sequel, the 
universal cover of a geometry $\Gamma$ of rank $n$ is its universal $(n-1)$-cover and we say that $\Gamma$ is simply connected if it is $(n-1)$-simply connected.

\subsection{The geometry far from a flag}

Suppose $\Delta$ is a thick building of connected spherical type and rank at least 2 . It is well known that, given a flag $F \neq \emptyset$ and a chamber $C$ of $\Delta$, there is a unique chamber $C_{F} \supseteq F$ at minimal distance from $C$ (Tits [18]). We denote the distance between $C$ and $C_{F}$ by $d(C, F)$. For every nonempty flag $X$, the distance $d(X, F)$ from $X$ to $F$ is the minimal distance $d(C, F)$ from $F$ to a chamber $C \supseteq X$. We say that a flag $X$ is far from $F$ if $d(X, F)$ is maximal, compatibly with the types of $F$ and $X$. We denote by $\operatorname{Far}_{\Delta}(F)$ the substructure of $\Delta$ formed by the elements far from $F$, with the incidence relation inherited from $\Delta$ but rectified as follows: two elements $x, y$ of $\operatorname{Far}_{\Delta}(F)$ are incident in $\operatorname{Far}_{\Delta}(F)$ if and only if they are incident in $\Delta$ and the flag $\{x, y\}$ is far from $F$.

As $\Delta$ is thick, the structure $\operatorname{Far}_{\Delta}(F)$ is firm. It is known that $\operatorname{Far}_{\Delta}(F)$ is residually connected (whence, it is a geometry) except for a few cases defined over $G F(2)$ (Blok and Brouwer [4]), but none of those exceptional cases will be met in this paper.

In the sequel we call $\operatorname{Far}_{\Delta}(F)$ a geometry of far away type, also a far away geometry, for short. Before to state the results of this paper, we mention a few examples, focusing on simple connectedness.

Example 1.1 Suppose $\Delta$ is a non-degenerate projective geometry of dimension $n \geq 3$ and let $A$ be a hyperplane or a point of $\Delta$. Then $\operatorname{Far}_{\Delta}(A)$ is an affine geometry or the dual of an affine geometry. In any case, $\operatorname{Far}_{\Delta}(A)$ is simply connected.

Example 1.2 With $\Delta$ as in Example 1, let $F$ be a point-hyperplane flag of $\Delta$. $\operatorname{Then}_{\operatorname{Far}}(F)$ is an affine-dual-affine geometry as in Van Nypelseer [19]. It follows from the main result of [19] that $\operatorname{Far}_{\Delta}(F)$ is simply connected.

Example 1.3 Let $\Delta$ be a thick polar space of rank $n>2$ and $p$ a point of $\Delta$. Then $\operatorname{Far}_{\Delta}(p)$ is an affine polar space (Cohen and Shult [7]). Affine polar spaces are simply connected

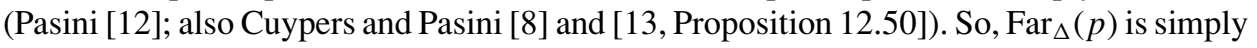
connected.

A similar conclusion holds when $\Delta$ is a building of type $D_{n}$ and $p$ is an element of $\Delta$ corresponding to a point of the polar space $\Pi$ associated to $\Delta$. Indeed, the subgeometry of $\Pi$ corresponding to $\operatorname{Far}_{\Delta}(p)$ is an affine polar space and, as recalled above, affine polar spaces are simply connected.

\subsection{Main results}

The geometries $\operatorname{Far}_{\Delta}(A), \operatorname{Far}_{\Delta}(F)$ and $\operatorname{Far}_{\Delta}(p)$ of Examples 1.1, 1.2 and 1.3 are simply connected. In this paper we obtain the same conclusion in a number of other cases. Explicitly, the following are the results we shall prove. 
Theorem 1.1 Let $\Delta$ be a non-degenerate projective geometry of dimension $n \geq 3$ and $A$ an element of $\Delta$ other than a point or a hyperplane. Then $\operatorname{Far}_{\Delta}(A)$ is simply connected.

Theorem 1.2 Let $\Delta$ be a thick polar space of rank $n \geq 3$ and $A$ a maximal singular subspace of $\Delta$. Namely $t(A)=n-1$, where the nonnegative integers $0,1,2, \ldots, n-1$ are taken as types, as follows:

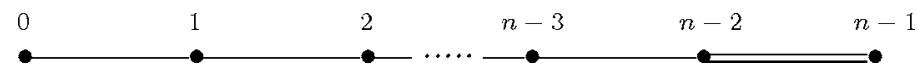

Then $\operatorname{Far}_{\Delta}(A)$ is simply connected, except when $n=3$ and $\Delta$ is either the symplectic variety $\mathcal{S}(5,2)$ of $P G(5,2)$ or the hermitean variety $\mathcal{H}(5,4)$ of $P G(5,4)$.

Theorem 1.3 For $n \geq 4$, let $\Delta$ be a thick building of type $D_{n}$ and $A$ an element corresponding to a maximal singular subspace of the polar space associated to $\Delta$. That is, $t(A)=+$ or - , where the nonnegative integers $0,1,2, \ldots, n-3$ and the symbols + and - are taken as types, as follows:

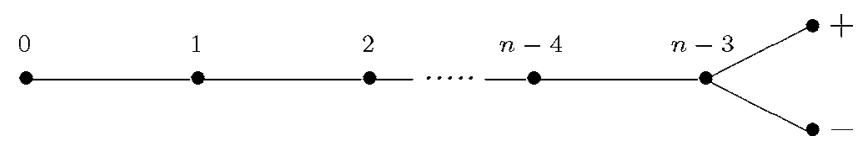

Then $\operatorname{Far}_{\Delta}(A)$ is simply connected.

Theorem 1.4 For $n \geq 4$, let $\Delta$ be a thick building of type $D_{n}$ and $F$ a flag of $\Delta$ of type $\{+,-\}$, with types as in Theorem 1.4. Then $\operatorname{Far}_{\Delta}(F)$ is simply connected except when $n=4$ and $\Delta$ is defined over $G F(2)$.

Theorem 1.5 Let $\Delta$ be a thick building of type $E_{6}$, with types $0,1,2,3,4,5$ as follows:

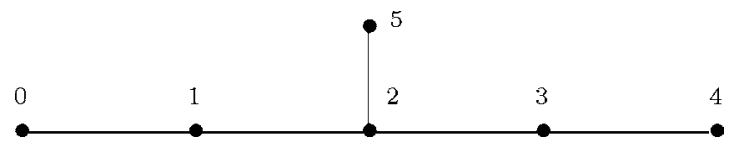

Then $\operatorname{Far}_{\Delta}(p)$ is simply connected for every 0-element $p$.

A few small cases are not covered by the previous theorems: Theorem 1.2 misses the cases of $\Delta=\mathcal{S}(5,2)$ and $\Delta=\mathcal{H}(5,4)$. Theorem 1.5 misses the case of $\Delta=D_{4}(2)$, the building of type $D_{4}$ over $G F(2)$. The next theorem settles those exceptions. 
Theorem 1.6 With $\Delta=\mathcal{S}(5,2), \mathcal{H}(5,4)$ or $D_{4}(2)$, let $X$ be a singular plane of $\Delta$ in the first two cases and $a\{+,-\}$-flag in the third case. In any case, let $\widetilde{\Gamma}$ be the universal cover of $\Gamma:=\operatorname{Far}_{\Delta}(X)$. Then,

(1) if $\Delta=\mathcal{S}(5,2)$, then $\widetilde{\Gamma}$ is a double cover;

(2) if $\Delta=\mathcal{H}(5,4)$, then $\widetilde{\Gamma}$ is a 4-fold cover;

(3) if $\Delta=D_{4}(2)$, then $\widetilde{\Gamma}$ is a double cover.

Claim (2) of the above theorem is new whereas (1) and (3) are known (Baumeister, Meixner and Pasini [2]). However, the proofs we will give of (1) and (3) are shorter than those of [2] and, we presume, more perspicuous.

We recall that the universal 2-cover and the universal cover of a geometry of rank 3 are the same thing, but in higher rank cases the former is possibly larger than the latter. Accordingly, a geometry of rank $n>3$ might be simply connected without being 2-simply connected. However, nearly all of the geometries considered in this paper are 2-simply connected. Explicitly:

Corollary 1.7 All far-away geometries considered in Theorems 1.1, 1.4 and 1.5 are 2simply connected. The geometries of Theorem 1.2 are 2-simply connected provided that $\Delta$ is neither the symplectic variety $\mathcal{S}(2 n-1,2)$ of $P G(2 n-1,2)$ nor the hermitean variety $\mathcal{H}(2 n-1,4)$ of $P G(2 n-1,4)$. The geometries of Theorem 1.4 are 2-simply connected provided their underlying field is different from $G F(2)$.

According to the above corollary, the following exceptional cases need a separate discussion:

(1) $\Gamma=\operatorname{Far}_{\Delta}(A)$ as in Theorem 1.2, but $\Delta=\mathcal{S}(2 n-1,2)$;

(2) $\Gamma=\operatorname{Far}_{\Delta}(A)$ as in Theorem 1.2 , but $\Delta=\mathcal{H}(2 n-1,4)$;

(3) $\Gamma=\operatorname{Far}_{\Delta}(F)$ as in Theorem 1.4, but $\Delta=D_{n}(2)$.

A description of the universal 2-cover $\widetilde{\Gamma}$ of $\Gamma$ is known in cases (1) and (3) (Baumeister, Meixner and Pasini [2]). In particular, $\widetilde{\Gamma}$ is a $2^{k}$-fold cover with $k=2^{n}-\left({ }^{n+1}\right)-1$ in case (1) and $k=2^{n-1}-\left(\begin{array}{c}n \\ 2\end{array}\right)-1$ in case (3). I will also offer a construction of $\widetilde{\Gamma}$ for cases (1) and (3) in Sections 9 and 10, which I think will clarify the descriptions given in [2]. A construction of $\widetilde{\Gamma}$ for $\Gamma$ as in case (2) is also given in Section 9. In principle, it is possible to exploit it to compute the size of $\widetilde{\Gamma}$ but, regretfully, I have been able to accomplish that computation only for $n \leq 4$ : When $n=3, \widetilde{\Gamma}$ is a 4-fold cover (Theorem 1.6(2)); when $n=4, \widetilde{\Gamma}$ is a $2^{8}$-fold cover.

As said at the beginning of this Introduction, I have not found the right approach to investigate $\operatorname{Far}_{\Delta}(x)$ with $\Delta$ a thick building of type $F_{4}$ and $x$ a point or a symp of $\Delta$. I only mention a partial result, proved in [16] (Corollary 9.9):

Result 1.8 Suppose $\Delta$ is of type $F_{4}(p)$ for a prime $p>2$ and $\operatorname{Res}(x) \cong \mathcal{S}(5, p)$. Then $\operatorname{Far}_{\Delta}(x)$ is 2-simply connected. 


\subsection{Organization of the paper}

The rest of this introduction contains some references and the list of the diagrams of the geometries of far-away type considered in the previous theorems.

A few general results and constructions to be used in this paper are recalled in Section 2. Sections 3, 4, 5 and 7 contain the proofs of Theorems 1.1, 1.2, 1.4 and 1.5 respectively. We will split the proof of Theorem 1.4 in two parts. The first part forms Section 6 and deals with the generic case, where either the underlying field $K$ of $\Delta$ is different from $G F(2)$ or $K=G F(2)$ but $\Delta$ has rank $n>5$. The case of $\Delta=D_{5}(2)$ remains to consider, but we pospone its discussion till Section 10. The proof of Corollary 1.7 is given in Section 8 . Claims (1) and (2) of Theorem 1.6 are proved in Section 9 and claim (3) is proved in Section 10. Universal 2-covers for the exceptional cases (1), (2) and (3) mentioned after Corollary 1.7 are discussed in Sections 9 and 10. The proof of Theorem 1.4 in the case of $\Delta=D_{5}(2)$, put aside in Section 6, will be obtained in Section 10 as a by-product of the informations we will collect in that section on the universal 2-cover of $\operatorname{Far}_{\Delta}(F)$.

\subsection{Remarks}

Remark 1.9 A few special cases of some of our theorems have been earlier discussed by a number of authors. For instance, Baumeister, Meixner and Pasini [2] consider the special case of Theorem 1.2 where $\Delta$ is the non-singular orthogonal quadric $\mathcal{Q}(2 n, 2)$ of $P G(2 n, 2)$. Baumeister, Shpectorov and Stroth [3] consider the case of $\Delta=\mathcal{Q}(2 n, q)$ for any $q$ (but their argument works as well for the general case of Theorem 1.2).

The special case of Theorem 1.4 with $\Delta$ defined over a finite field is implicit in Munemasa and Shpectorov [10] and Munemasa, Pasechnik and Shpectorov [11] (also in Hybrechts and Pasini [9] when the underlying field of $\Delta$ is $G F(2))$.

When $K=G F(q)$ with $q>2$, the conclusion of Theorem 1.4 is contained in Baumeister and Stroth [1], who obtained that result group-theoretically, whereas the case of $q=2$ is discussed by Baumeister, Meixner and Pasini [2].

Turning to $\operatorname{Far}_{\Delta}(A)$ with $\Delta=\mathcal{H}(5,4)$, let $\mathcal{H}$ be the collinearity graph of the dual of $\Gamma:=\operatorname{Far}_{\Delta}(A)$, having the planes and the lines of $\Gamma$ as vertices and edges. Then $\mathcal{H}$ is isomorphic to the hermitean forms graph over $G F(4)$. A quadruple cover of $\mathcal{H}$ is described by Brouwer, Cohen and Neumaier [5, p. 365]. That cover is in fact the collinearity graph of the dual of the universal cover of $\Gamma$. A double cover of $\mathcal{H}$ is also described in [5]. It corresponds to a (non simply connected) double cover of $\Gamma$.

Remark 1.10 The far-away geometries considered in the previous theorems belong to the following diagrams, where • Af $\bullet$ stands for the class of affine planes, $\bullet$ Af* is the class of dual affine planes and Af represents the class of affine generalized quadrangles, which are geometries obtained from generalized quadrangles by removing a maximal full subquadrangle, an ovoid or the star of a point (the latter is always the case here). In all cases but Theorem 1.1 diagrams are given the orientation opposite to that used in the statements of the previous theorems. In the first picture, $k:=n-1-d$ and $d=t(A)$ is the projective dimension of $A$. 


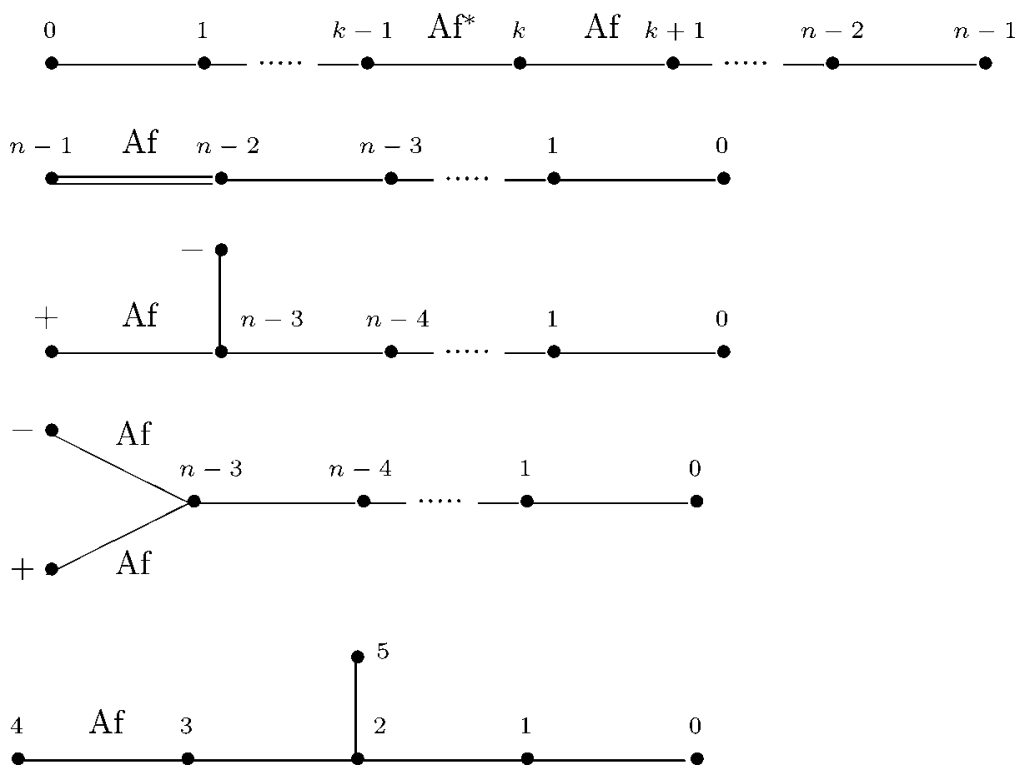

Remark 1.11 In the case considered in Theorem 1.1, if $k=n-1-t(A)$ as above, then the $\{0,1, \ldots, k-1\}$-truncation of $\operatorname{Far}_{\Delta}(A)$ is an attenuated space. So, Theorem 1.1 implies that attenuated spaces of rank at least 3 are simply connected.

\section{A selection of general results}

\subsection{Terminology and notation}

As in [13], given an element $x$ of a geometry $\Gamma$, we denote the type of $x$ by $t(x)$, but we change the notation of [13] for residues, denoting the residue of $x$ by $\operatorname{Res}_{\Gamma}(x)$ (also $\operatorname{Res}(x)$ if no ambiguity arises). The same notation will be used for flags. As in [13], given a subset $J \neq \varnothing$ of the type-set $I$ of $\Gamma$, we denote by $\operatorname{Tr}_{J}^{-}(\Gamma)$ the geometry obtained from $\Gamma$ by removing all elements of type $j \in J$. We call $\operatorname{Tr}_{J}^{-}(\Gamma)$ the $J$-truncation of $\Gamma$. When $J \subset I$, we set $\operatorname{Tr}_{J}^{+}(\Gamma):=\operatorname{Tr}_{I \backslash J}^{-}(\Gamma)$ and we call it the $J$-cotruncation of $\Gamma$.

As in [13], we denote by $\mathcal{D}(\Gamma)$ the diagram graph of $\Gamma$ (also called basic diagram of $\Gamma)$. Suppose that $\mathcal{D}(\Gamma)$ is connected. Denoted by $I$ the set of types of $\Gamma$ and given a type $0 \in I$, let $\operatorname{fr}(0)$ be the neighbourhood of 0 in $\mathcal{D}(\Gamma)$. The 0 -point-line system $\mathcal{L}_{0}(\Gamma)$ of $\Gamma$ is the point-line geometry having the 0 -elements of $\Gamma$ as points and the flags of type $\operatorname{fr}(0)$ as lines, with the incidence relation inherited from $\Gamma$. The collinarity graph of $\mathcal{L}_{0}(\Gamma)$ will be denoted by $\mathcal{G}_{0}(\Gamma)$.

\subsection{A criterion for simple connectedness}

Given a flag $F \neq \emptyset$ with $0 \notin t(F)$, $\operatorname{Res}(F)$ is the direct sum of subgeometries corresponding to the connected components of the graph induced by $\mathcal{D}(\Gamma)$ on $I \backslash t(F)$. In particular, denoted 
by $I_{0}$ the connected component of 0 in that induced subgraph, the $I_{0}$-cotruncation of $\operatorname{Res}(F)$ is a direct summand of $\operatorname{Res}(F)$. We denote it by $\operatorname{Res}_{0}(F)$. When $\operatorname{fr}(0) \nsubseteq t(F), \operatorname{Res}_{0}(F)$ has rank at least 2 and we can consider its 0 -point-line system $\mathcal{L}_{0}\left(\operatorname{Res}_{0}(F)\right)$. We denote the collinearity graph of $\mathcal{L}_{0}\left(\operatorname{Res}_{0}(F)\right)$ by $\mathcal{G}_{0}(F)$. When $\operatorname{fr}(0) \subseteq t(F)$, then $\operatorname{Res}_{0}(F)$ has rank 1 (in fact, it is the point-set of a line of $\mathcal{L}_{0}(\Gamma)$ ). In that case $\mathcal{G}_{0}(F)$ stands for the complete graph over the set $\operatorname{Res}_{0}(F)$.

We say that a closed path of $\mathcal{G}_{0}(\Gamma)$ is good if it is a path of $\mathcal{G}_{0}(F)$ for some nonempty flag $F$. The following proposition, which immediately follows from [13, Theorem 12.64], is the main tool we will use in this paper:

Proposition 2.1 Suppose that no two lines of $\mathcal{L}_{0}(\Gamma)$ meet in more than one point. Then $\Gamma$ is simply connected if and only if every closed path of $\mathcal{G}_{0}(\Gamma)$ splits in good closed paths.

\subsection{Universal covers of shadow geometries}

The geometry $\mathcal{L}_{0}(\Gamma)$ coincides with the $\{0,1\}$-cotruncation of a geometry of the same rank as $\Gamma$ but with a string as its diagram graph, usually called the 0-shadow geometry of $\Gamma$ and denoted by $\mathrm{Sh}_{0}(\Gamma)$ (but we warn that the symbol $\mathrm{Gr}_{0}(\Gamma)$ and the words grassmann geometry are used in [13] instead of $\mathrm{Sh}_{0}(\Gamma)$ and shadow geometry). We are not going to recall the construction of $\mathrm{Sh}_{0}(\Gamma)$ in general. We only consider the special case where $\Gamma$ belongs to a diagram as follows, where $\mathcal{X}_{1}, \mathcal{X}_{2}, \ldots, \mathcal{X}_{n-3}$ and $\mathcal{Y}$ are classes of geometries of rank 2 different from generalized digons and $0,1, \ldots, n-3,+$ and - are the types:

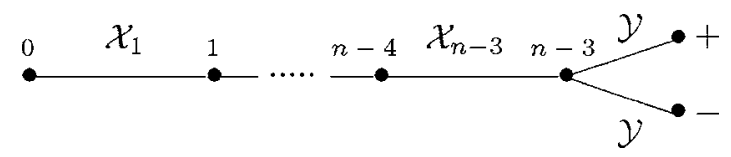

In this case the definition of $\operatorname{Sh}_{0}(\Gamma)$ is a straightforward generalization of the construction of the polar space associated to a $D_{n}$-building. The elements of $\operatorname{Sh}_{0}(\Gamma)$ are the elements and the $\{+,-\}$-flags of $\Gamma$. The elements of $\Gamma$ of type $i=0,1, \ldots, n-3$ keep their type in $\operatorname{Sh}_{0}(\Gamma)$, those of type + and - form the class of $(n-1)$-elements of $\operatorname{Sh}_{0}(\Gamma)$ and the $\{+,-\}$-flags are given the type $n-2$ as elements of $\operatorname{Sh}_{0}(\Gamma)$. The incidence relation of $\mathrm{Sh}_{0}(\Gamma)$ is inherited from $\Gamma$, except that two elements of type + and - are never incident in $\mathrm{Sh}_{0}(\Gamma)$. The geometry $\mathrm{Sh}_{0}(\Gamma)$ belongs to the following diagram:

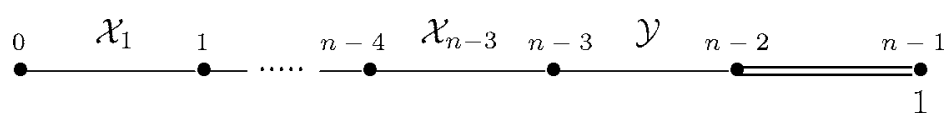

Note that the residues of $\operatorname{Sh}_{0}(\Gamma)$ of type $\{n-2, n-1\}$ are grids, as the order 1 at the right end of (2) reminds us.

The following are contained in [14, Theorems 7 and 9] (see also Rinauro [17]):

Proposition 2.2 Let $\Gamma$ be a geometry belonging to diagram (1). Then the universal cover of $\mathrm{Sh}_{0}(\Gamma)$ is the 0 -shadow geometry of the universal cover of $\Gamma$. Suppose furthermore that 
$\mathrm{Sh}_{0}(\Gamma)$ admits the universal 2-cover. Then $\Gamma$ also admits the universal 2-cover and the universal 2-cover of $\mathrm{Sh}_{0}(\Gamma)$ is the 0-shadow geometry of the universal 2-cover of $\Gamma$.

Remark 2.3 If $\mathcal{C}$ is the chamber system of a geometry $\Gamma$ of rank $n>3$, we do not know if the universal 2-cover of $\mathcal{C}$ necessarily comes from a geometry. When it does, then that geometry is the universal 2-cover of $\Gamma$ and we may say that $\Gamma$ admits the universal 2-cover. This remark explains why the existence of the universal 2-cover of $\operatorname{Sh}_{0}(\Gamma)$ is put as an hypothesis in the second part of Proposition 2.2. On the other hand, every geometry admits the universal cover. So, no hypothesis like that is needed in the first part. We could drop it from the second part too by rephrasing that statement in terms of chamber systems, but we prefer not to concern ourselves with them in this paper. Anyhow, all geometries considered in this paper admit the universal 2-cover. Indeed, for each of them, either we prove that it is 2-simply connected, or we construct its universal 2-cover as a geometry.

\subsection{Expansions of $G F(2)$-embeddings of matroids}

In this section we discuss a special case of the theory of embeddings and expansions of [16], taking $G F(2)$-vector spaces as codomains for the considered embeddings and assuming that the geometries to embed are finite dimensional simple matroids (also called dimensional linear spaces; see Buekenhout [6, Chapter 6]).

Henceforth $\mathcal{M}$ is a given simple matroid of finite dimension $n \geq 1$, regarded as an $n$-tuple $\mathcal{M}=\left(P, \mathcal{F}_{1}, \ldots, \mathcal{F}_{n-1}\right)$, where $P$ is the set of points and, for $i=1,2, \ldots, n-1, \mathcal{F}_{i}$ is the set of $i$-dimensional flats, also called $i$-flats for short. In particular, the 1 -flats are the lines of $\mathcal{M}$. We set $\mathcal{L}:=\mathcal{F}_{1}$ and $\mathcal{F}:=\cup_{i=1}^{n-1} \mathcal{F}_{i}$.

Given a vector space $V$ over $G F(2)$, a $G F(2)$-embedding $\varepsilon: \mathcal{M} \rightarrow V$ of $\mathcal{M}$ in $V$ is an injective mapping $\varepsilon: P \rightarrow V \backslash\{0\}$ such that $\varepsilon(P)$ spans $V$ and, for any two flats $X, Y \in \mathcal{F}$, we have $\langle\varepsilon(X)\rangle \subseteq\langle\varepsilon(Y)\rangle$ only if $X \subseteq Y$ (where, regarding $X$ as a subset of $P$, we write $\varepsilon(X)$ for $\left.\{\varepsilon(p)\}_{p \in X}\right)$.

For $X \in \mathcal{F}$, we put $V_{X}:=\langle\varepsilon(X)\rangle$. Also, for $x \in P$, we denote by $V_{x}$ the 1-dimensional subspace of $V$ spanned by $\varepsilon(x)$.

The expansion $\operatorname{Exp}(\varepsilon)$ of $\varepsilon$ is the geometry of rank $n+1$ defined as follows: The types of $\operatorname{Exp}(\varepsilon)$ are the integers $0,1, \ldots, n$; the vectors of $V$ are the elements of $\operatorname{Exp}(\varepsilon)$ of type 0 , which we also call points of $\operatorname{Exp}(\varepsilon)$; the elements of type 1 are the cosets in $V$ of the 1-dimensional subspaces $V_{x}$ for $x \in P$ and the elements of type $j=2,3, \ldots, n$ are the cosets of the subspaces $V_{X}$ in $V$, for $X \in \mathcal{F}_{j-1}$; the incidence relation of $\operatorname{Exp}(\varepsilon)$ is the natural one, namely symmetrized inclusion.

Clearly, $\operatorname{Res}(v) \cong \mathcal{M}$ for every point $v \in V$ of $\operatorname{Exp}(\varepsilon)$ and, given an element $W=v+V_{X}$ of $\operatorname{Exp}(\varepsilon)$ of type $j>1$, the $\{0,1, \ldots, j-1\}$-cotruncation $\operatorname{Res}_{0}(W)$ of $\operatorname{Res}(W)$ is isomorphic to $\operatorname{Exp}\left(\varepsilon_{X}\right)$, where $\varepsilon_{X}: \operatorname{Res}_{0}(X) \rightarrow V_{X}$ is the restriction of $\varepsilon$ to $X$. In particular, if all lines of $\mathcal{M}$ have finite size $s>2$, then the $\{0,1\}$-residues of $\operatorname{Exp}(\varepsilon)$ are quotients of the point-graph of the $s$-dimensional cube; explicitly, if $d=\operatorname{dim}\left(V_{L}\right)$ for $L \in \mathcal{L}$, then $\operatorname{Res}_{0}\left(v+V_{L}\right)$ is a $2^{s-d}$-fold quotient of that graph. If every line of $\mathcal{M}$ has just two points, then the $\{0,1\}-$ residues of $\operatorname{Exp}(\varepsilon)$ are ordinary quadrangles. 
Example 2.1 Assume that the lines of $\mathcal{M}$ have size 3. If $\operatorname{dim}\left(V_{L}\right)=3$ for every $L \in \mathcal{L}$, then the $\{0,1\}$-residues of $\operatorname{Exp}(\varepsilon)$ are isomorphic 3-dimensional cubes. If $\operatorname{dim}\left(V_{L}\right)=2$ for every line $L$, then those residues are isomorphic to $A G(2,2)$, which may be regarded as the quotient of the 3 -cube by the antipodal relation.

Example 2.2 Suppose that, for every $L \in \mathcal{L},|L|=5, \operatorname{dim}\left(V_{L}\right)=4$ and $\left\{V_{x}\right\}_{x \in L}$ is an ovoid of $P G\left(V_{L}\right) \cong P G(3,2)$ (compare Section 9, Lemma 9.4). Then the $\{0,1\}$-residues of $\operatorname{Exp}(\varepsilon)$ are isomorphic to the quotient of the 5-cube by the antipodal relation.

Let $\widehat{V}$ be a $|P|$-dimensional vector space over $G F(2)$ and $\iota$ a given bijection from $P$ to a basis of $\widehat{V}$. Clearly, $\iota$ is a $G F(2)$-embedding of $\mathcal{M}$. We call it the free $G F(2)$-embedding of $\mathcal{M}$.

Every $G F(2)$-embedding of $\mathcal{M}$ is involved in the free embedding. More explicitly, given a $G F(2)$-embedding $\varepsilon: \mathcal{M} \rightarrow V$, the mapping sending the image $\iota(x)$ of a point $x \in P$ to the vector $\varepsilon(x) \in V$, extends to a surjective linear transformation $\pi: \widehat{V} \rightarrow V$. As $\pi$ maps $\iota(X)$ onto $\varepsilon(X)$, it maps the subspace $\widehat{V}_{X}:=\langle\iota(X)\rangle$ of $\widehat{V}$ onto the subspace $V_{X}$ of $V$ and, denoted by $K$ the kernel of $\pi$ and by $K_{X}$ the kernel of the restriction of $\pi$ to $\widehat{V}_{X}$, we have

$$
K_{X}=K \cap \widehat{V}_{X}
$$

Henceforth we assume $n \geq 2$ and define:

$$
\widetilde{K}:=\left\langle K_{X}\right\rangle_{X \in \mathcal{F}}, \quad \widetilde{K}^{(1)}:=\left\langle K_{L}\right\rangle_{L \in \mathcal{L}}, \quad \widetilde{V}:=\widehat{V} / \widetilde{K}, \quad \widetilde{V}^{(1)}:=\widehat{V} / \widetilde{K}^{(1)} .
$$

We call $\widetilde{K}$ and $\widetilde{K}^{(1)}$ the local kernel and the 1-local kernel of $\varepsilon$. The subspace $K$ will be called the global kernel of $\varepsilon$.

Clearly, $\widetilde{K}^{(1)} \leq \widetilde{K} \leq K$ and $\pi=\tilde{\varphi} \tilde{\pi}=\tilde{\varphi}^{(1)} \tilde{\pi}^{(1)}$ where $\tilde{\pi}$ and $\tilde{\pi}^{(1)}$ are the natural projections of $\widehat{V}$ onto $\widetilde{V}$ and $\widetilde{V}^{(1)}$, and $\tilde{\varphi}$ and $\tilde{\varphi}^{(1)}$ are the natural projections of $\widetilde{V}$ and $\widetilde{V}^{(1)}$ onto $V$. Therefore,

$$
\begin{aligned}
\widetilde{K} \cap \widehat{V}_{X}=K_{X} & \text { for all } X \in \mathcal{F}, \\
\widetilde{K}^{(1)} \cap \widehat{V}_{L}=K_{L} & \text { for all } L \in \mathcal{L} .
\end{aligned}
$$

Lemma 2.4 Regarded $\widetilde{V}, \widetilde{V}^{(1)}$ and the subspaces $V_{X}$ of $V$ as additive groups and the posets

$$
\mathcal{A}:=\left(\left\{V_{X}\right\}_{X \in \mathcal{F}} \cup\left\{V_{x}\right\}_{x \in P}, \subseteq\right), \quad \mathcal{A}^{(1)}:=\left(\left\{V_{L}\right\}_{L \in \mathcal{L}} \cup\left\{V_{x}\right\}_{x \in P}, \subseteq\right)
$$

as amalgams of groups, $\widetilde{V}$ is the universal completion of $\mathcal{A}$ and $\widetilde{V}^{(1)}$ is the universal completion of $\mathcal{A}^{(1)}$.

Proof: Let $U$ be the universal completion of $\mathcal{A}$. As all groups $V_{X}$ are generated by involutions, $U$ is generated by involutions. Furthermore, as the points of $\mathcal{M}$ are mutually collinear, any two generating involutions of $U$ are contained in $V_{L}$ for some $L \in \mathcal{L}$. So, 
they commute in $V_{L}$. Hence $U$ is an elementary abelian 2-group. It is now clear that $U$ is a homomorphic image of $\widehat{V}$. The equality $U=\widetilde{V}$ follows from (2). By a similar argument, except for using (3) instead of (2), one can prove that $\widetilde{V}^{(1)}$ is the universal completion of $\mathcal{A}^{(1)}$.

We define the hull $\tilde{\varepsilon}$ and the $1-h u l l \tilde{\varepsilon}^{(1)}$ of $\varepsilon$ as the compositions of the free embedding $\iota$ with the projections $\tilde{\pi}: \widehat{V} \rightarrow \widetilde{V}$ and $\tilde{\pi}^{(1)}: \widehat{V} \rightarrow \widetilde{V}^{(1)}$ :

$$
\tilde{\varepsilon}:=\tilde{\pi} \iota, \quad \tilde{\varepsilon}^{(1)}:=\tilde{\pi}^{(1)} \iota .
$$

Both $\tilde{\varepsilon}$ and $\tilde{\varepsilon}^{(1)}$ are $G F(2)$-embeddings and we have $\varepsilon=\tilde{\varphi} \tilde{\varepsilon}=\tilde{\varphi}^{(1)} \tilde{\varepsilon}^{(1)}$. Also, $\tilde{\pi}=\psi \tilde{\pi}^{(1)}$ and $\tilde{\varphi}^{(1)}=\tilde{\varphi} \psi$, where $\psi$ is the natural projection of $\tilde{V}^{(1)}$ onto $\widetilde{V}$. Hence $\tilde{\varepsilon}=\psi \tilde{\varepsilon}^{(1)}$.

Proposition 2.5 The geometries $\operatorname{Exp}(\tilde{\varepsilon})$ and $\operatorname{Exp}\left(\tilde{\varepsilon}^{(1)}\right)$ are respectively the universal cover and the universal 2-cover of $\operatorname{Exp}(\varepsilon)$. Their deck groups are isomorphic to $K / \widetilde{K}$ and $K / \widetilde{K}^{(1)}$, respectively.

Proof: The claims on $\operatorname{Exp}(\tilde{\varepsilon})$ and $K / \widetilde{K}$ follow from Theorem 3.3 of [16] and the fact that $\widetilde{V}$ is the universal completion of $\mathcal{A}$ (Lemma 2.4$)$. The claims on $\operatorname{Exp}\left(\tilde{\varepsilon}^{(1)}\right)$ and $K / \widetilde{K}^{(1)}$ follow from Theorem 4.4 of [16], the fact that $\widetilde{V}^{(1)}$ is the universal completion of $\mathcal{A}^{(1)}$ and the well known fact that matroids are 2-simply connected.

Corollary 2.6 The geometry $\operatorname{Exp}(\varepsilon)$ is simply connected if and only if $K=\widetilde{K}$. It is 2-simply connected if and only if $K=\widetilde{K}^{(1)}$.

(Trivial, by the second part of Proposition 2.5.) Corollary 2.6 is also helpful to compute $\widetilde{K}$ in certain cases. Note first that, according to (1), for $X, Y \in \mathcal{F}$ we have $K_{X} \subseteq K_{Y}$ whenever $X \subseteq Y$. Therefore, in any case:

$$
\widetilde{K}=\left\langle K_{S}\right\rangle_{S \in \mathcal{F}_{n-1}}
$$

Corollary 2.7 Suppose that, for a given $k>1$ and every $i=k, k+1, \ldots, n-1$, all $\{0,1, \ldots, i\}$-residues of $\operatorname{Exp}(\varepsilon)$ are simply connected. Then

$$
\widetilde{K}=\left\langle K_{S}\right\rangle_{S \in \mathcal{F}_{k-1}} .
$$

In particular, if all residues of $\operatorname{Exp}(\varepsilon)$ of type $\{0,1, \ldots, i\}$ are simply connected for all $i=2,3, \ldots, n-1$, then $\widetilde{K}=\widetilde{K}^{(1)}$.

Proof: As previously remarked, given an $(i+1)$-element $W=v+V_{X}$ of $\operatorname{Exp}(\varepsilon)$, the $\{0,1, \ldots, i\}$-cotruncation of $\operatorname{Res}(W)$ is isomorphic to the expansion of the restriction $\varepsilon_{X}$ of $\varepsilon$ to $X$. The conclusion follows by induction, applying (4) and Corollary 2.6 to $K_{X}$, which is the global kernel of $\varepsilon_{X}$.

Proposition 2.8 We have $\tilde{\varepsilon}^{(1)}=\iota$ if and only if the set $\varepsilon(L)$ is linearly independent for every $L \in \mathcal{L}$. 
Proof: The set $\varepsilon(L)$ is independent if and only if $K_{L}=0$. The conclusion immediately follows from this remark.

Corollary 2.9 If all lines of $\mathcal{M}$ have size 2 , then $\tilde{\varepsilon}^{(1)}=\iota$.

Proof: If $|L|=2$, then $\varepsilon(L)$ only contains two vectors, whence it is independent. The conclusion follows from Proposition 2.8.

\section{Proof of Theorem 1.1}

Let $d=\operatorname{dim}(A)$. By assumption, $0<d<n-1$. We will apply Proposition 2.1 with the points of $\Gamma=\operatorname{Far}_{\Delta}(A)$ taken as 0 -elements. So, the lines of $\mathcal{L}_{0}(\Gamma)$ are the lines of $\Delta$ that miss $A$. We call them lines of $\Gamma$, for short.

Lemma 3.1 For every point $p$ and every line $L$ of $\Gamma$, at most one of the points of $L$ is non-collinear with $p$ in $\Gamma$.

Proof: If $p \in L$ there is nothing to prove. Suppose $p \notin L$ and let $\pi$ be the plane of $\Delta$ spanned by $p$ and $L$. As $L \cap A=\emptyset$, we have $|\pi \cap A| \leq 1$. Hence at most one of the lines of $\pi$ through $p$ meets $A$.

Lemma 3.2 The graph $\mathcal{G}_{0}(\Gamma)$ has diameter 2 and every closed path of $\mathcal{G}_{0}(\Gamma)$ splits in triangles.

Proof: Let $(a, b, c, d)$ be a path of $\mathcal{G}_{0}(\Gamma)$ with $a \ngtr c$ and $b \ngtr d$, where $\sim$ stands for the collinearity relation of $\Gamma$. Denoted by $L$ the line of $\Gamma$ through $c$ and $d$, we have $L \backslash\{c, d\} \subseteq a^{\sim} \cap b^{\sim}$ by Lemma 3.1. However, $L \backslash\{c, d\} \neq \emptyset$, as $|L| \geq 3$. Thus, there exists a point collinear with all of $a, b, c, d$. Both claims of the corollary follow from this remark.

Lemma 3.3 Every bad triangle of $\mathcal{G}_{0}(\Gamma)$ splits in good triangles.

Proof: Every triangle of $\mathcal{G}_{0}(\Gamma)$ is contained in plane of $\Delta$. Furthermore, if a plane of $\Delta$ contains a line of $\Gamma$, then it has at most one point in common with $A$. Thus, all planes containing triangles of $\mathcal{G}_{0}(A)$ meet $A$ in at most a point.

When $n-d=2$, a plane $P$ of $\Delta$ belongs to $\Gamma$ if and only if $P \cap A$ is a point. When $n-d>2, P$ belongs to $\Gamma$ if and only if $P \cap A=\emptyset$. So, when $n-d=2$ all triangles of $\mathcal{G}_{0}(\Gamma)$ are contained in planes of $\Gamma$, hence all of them are good.

Assume $n-d>2$. Let $\{a, b, c\}$ be a bad triangle of $\mathcal{G}_{0}(\Gamma)$ and $P=\langle a, b, c\rangle$ the plane of $\Delta$ spanned by it. Then $P \cap A$ is a point, say $p$. However, as $n-d>2, P$ is contained in a 3-space $S$ such $S \cap A=p$. Clearly, given a point $x \in S \backslash P$, each of the planes $\langle a, b, x\rangle$, $\langle b, c, x\rangle$ and $\langle a, b, x\rangle$ misses $A$. So, each of the triangles $\{a, b, x\},\{b, c, x\}$ and $\{a, b, x\}$ is good.

Theorem 1.1 follows from Lemmas 3.2 and 3.3 via Proposition 2.1. 


\section{Proof of Theorem 1.2}

As noticed in Remark 1.9, a proof of Theorem 1.2 is given by Baumeister, Shpectorov and Stroth [3]. However, as [3] has not yet appeared, we will prove that theorem here, for the sake of completeness. Our proof is in fact very similar to that of [3].

The elements of $\Gamma:=\operatorname{Far}_{\Delta}(A)$ are the points of $\Delta$ that do not belong to $A$ and the singular subspaces $S$ of $\Delta$ such that $S \cap A=\emptyset$. We keep for the elements of $\Gamma$ the types they have in $\Delta$ and their usual names, as point, line, plane, maximal singular subspace. We denote by $\sim$ the collinearity relation of $\Gamma$ keeping the symbol $\perp$ for the collinearity relation of $\Delta$. If $a, b$ are distinct collinear points of $\Delta$, we denote by $\langle a, b\rangle$ the line of $\Delta$ through them.

\subsection{Preliminaries}

If $\{a, b, c\}$ is a triangle of $\mathcal{G}_{0}(\Gamma)$ not contained in a line, then $\{a, b, c\}$ is contained in a unique plane $\langle a, b, c\rangle$ of $\Delta$. The triangle $\{a, b, c\}$ is good if and only if $\langle a, b, c\rangle \cap$ $A=\emptyset$.

Lemma 4.1 If $\{a, b, c\}$ is a bad triangle of $\mathcal{G}_{0}(\Gamma)$, then $\langle a, b, c\rangle \cap A$ is a point.

Proof: The plane $\langle a, b, c\rangle$ contains three lines of $\Gamma$, namely three lines of $\Delta$ that miss $A$. Thus, $\langle a, b, c\rangle \cap A$ cannot contain any line.

Lemma 4.2 Given in $\Gamma$ a maximal singular subspace $S$ and a point $p$, then $p^{\sim}$ contains all points of $p^{\perp} \cap S$ but at most one.

Proof: Let $S^{\prime}$ be the maximal singular subspace of $\Delta$ spanned by $\{p\} \cup\left(p^{\perp} \cap S\right)$. Then $A \cap\left(p^{\perp} \cap S\right)=\emptyset$, as $S \cap A=\emptyset$. However, $p^{\perp} \cap S$ is a hyperplane of $S^{\prime}$. Hence $S^{\prime} \cap A$ contains at most one point. Consequently, at most one of the lines of $S^{\prime}$ through $p$ is missing in $\Gamma$.

Lemma 4.3 The graph $\mathcal{G}_{0}(\Gamma)$ has diameter 2 .

Proof: Given two points $a, b$ of $\Gamma$, let $S$ be a maximal singular subspace of $\Gamma$ containing $b$. If $a \in S$, then $a \sim b$. Otherwise, $a^{\sim} \cap S \neq \emptyset$ by Lemma 4.2. Picked a point $c \in a^{\sim} \cap S$, we have $a \sim c \sim b$.

Lemma 4.4 Every closed path of $\mathcal{G}_{0}(\Gamma)$ splits in quadrangles and triangles.

Proof: By Lemma 4.3, every closed path of $\mathcal{G}_{0}(\Gamma)$ splits in pentagons, quadrangles and triangles. We shall prove that every pentagon splits in quadrangles and triangles.

Given five points $a_{0}, a_{1}, a_{2}, a_{3}, a_{4}$ of $\Gamma$ with $a_{i} \sim a_{i+1}$ for $0 \leq i \leq 4$ (indices computed modulo 5), pick a maximal singular subspace $S$ of $\Gamma$ on the line $\left\langle a_{2}, a_{3}\right\rangle$. By Lemma 4.2 , 
$a_{0}^{\sim} \cap S$ contains at least one point, say $b$. Thus, the pentagon $\left\{a_{0}, a_{1}, a_{2}, a_{3}, a_{4}\right\}$ splits in $\left\{a_{0}, a_{1}, a_{2}, b\right\},\left\{a_{0}, a_{4}, a_{3}, b\right\}$ and $\left\{a_{2}, a_{3}, b\right\}$.

So far, we have not distinguished between the cases of $n>3$ and $n=3$, but from now on we must discuss them separately.

\subsection{The case of $n>3$}

Suppose $\Delta$ has rank $n>3$.

Lemma 4.5 Every quadrangle of $\mathcal{G}_{0}(\Gamma)$ splits in triangles.

Proof: Given four points $a_{0}, a_{1}, a_{2}, a_{3}$ of $\Gamma$ with $a_{i} \sim a_{i+1}$ for $i=0,1,2,3$ (indices computed modulo 4 ), pick a maximal singular subspace $S$ of $\Gamma$ containing $\left\langle a_{1}, a_{2}\right\rangle$. The sets $a_{0}^{\perp} \cap S$ and $a_{3}^{\perp} \cap S$ are hyperplanes in the projective geometry $\operatorname{Res}(S)$. Hence $a_{0}^{\perp} \cap a_{3}^{\perp} \cap S$ contains at least one line $L$, as $\operatorname{dim}(S)=n-1 \geq 3$. Clearly, $L$ belongs to $\Gamma$, as $S \in \Gamma$. By Lemma 4.2, for $i=0,3$ at most one point of $L$ is missing in $a_{i}^{\sim} \cap S$. Hence $L$ contains at least one point $b \in a_{0}^{\sim} \cap a_{3}^{\sim}$. Thus, $\left\{a_{0}, a_{1}, a_{2}, a_{3}\right\}$ splits in four triangles, namely the triangles $\left\{a_{i}, a_{i+1}, b\right\}$ for $i=0,1,2,3$.

Lemma 4.6 Every bad triangle of $\mathcal{G}_{0}(\Gamma)$ splits in good triangles.

Proof: Let $\{a, b, c\}$ be a bad triangle. By Lemma 4.1, $\langle a, b, c\rangle$ meets $A$ in one point $p$. Note that $p$ does not belong to any of the lines $\langle a, b\rangle,\langle b, c\rangle$ or $\langle c, a\rangle$ as all these lines belong to $\Gamma$. Let $S$ be a maximal singular subspace of $\Gamma$ containing $\langle b, c\rangle$. Then $a \notin S$. Consequently, $a^{\perp} \cap S$ is an (n-2)-dimensional subspace of $S$. However, only one point of $a^{\perp} \cap S$ is missing in $a^{\sim}$ and, as $p \notin \Gamma$, that point is necessarily the intersection of $\langle b, c\rangle$ with the line $\langle a, p\rangle$ of $\Delta$. As $\operatorname{dim}\left(a^{\perp} \cap S\right)=n-2 \geq 2, a^{\sim} \cap S$ contains two lines $L, M$ containing $b$ and $c$ respectively and meeting in a point $d$. Clearly, each of the triangles $\{a, b, d\},\{a, c, d\}$ and $\{b, c, d\}$ is good.

The simple connectedness of $\Gamma$ follows from Lemmas 4.4, 4.5 and 4.6 via Proposition 2.1.

\subsection{The case of $n=3$}

In this subsection, $n=3$ and $\Delta$ is neither $\mathcal{S}(5,2)$ nor $\mathcal{H}(5,4)$. Namely, every line of $\Delta$ belongs to at least four planes. Given a plane $P$ of $\Delta$ and a point or a line $X$, we denote by $p_{P}(X)$ the plane of $\Delta$ containing $X \cup\left(X^{\perp} \cap P\right)$.

Lemma 4.7 Given a line $L$ of $\Gamma$, all but one of the planes of $\Delta$ on $L$ belong to $\Gamma$. The missing plane meets $A$ in one point.

Proof: The plane $p_{A}(L)$ is the missing one. Indeed $L^{\perp} \cap A$ is a point and that point together with $L$ span $p_{A}(L)$. 
Lemma 4.8 Given in $\Gamma$ a line $L$ and a point $p \notin L$, one of the following occurs:

(1) $p$ and $L$ are coplanar in $\Gamma$.

(2) $p^{\sim} \cap L=L \backslash\{x\}$ for a point $x \in L$. In this case $p^{\sim} \cap P=L \backslash\{x\}$ for every plane $P \in \operatorname{Res}_{\Gamma}(L)$.

(3) $p^{\sim} \cap L=\{x\}$ for a point $x \in L$. In this case there exists at most one plane $P_{0} \in \operatorname{Res}_{\Gamma}(L)$ such that $p^{\sim} \cap P_{0}=M \backslash\left\{x_{0}\right\}$ for a line $M$ of $P_{0}$ on $p$ and a point $x_{0} \in M$. If $P$ is any of the remaining planes of $\operatorname{Res}_{\Gamma}(L)$, then $p^{\sim} \cap P$ is a line through $x$.

(4) $p^{\sim} \cap L=\emptyset$. In this case $p^{\perp} \cap L$ is a point, say $x$, and for every plane $P \in \operatorname{Res}_{\Gamma}(L)$, we have $p^{\sim} \cap P=M \backslash\{x\}$ for a line $M$ of $P$ on $x$.

Proof: If $p$ and $L$ are coplanar in $\Delta$, then we have either (1) or (2) according to whether the plane of $\Delta$ containing $L$ and $p$ belongs to $\Gamma$ or not (see Lemma 4.7 for the latter case). Suppose $p^{\perp} \cap L$ is a point, say $x$, and put $L^{\prime}:=\langle p, x\rangle$.

If $L^{\prime} \in \Gamma$ then we are in case (3). Given two planes $P_{1}, P_{2} \in \operatorname{Res}_{\Gamma}(L)$, let $M_{i}:=p^{\perp} \cap P_{i}$ and suppose that $p^{\sim} \cap P_{i}$ misses a point $x_{i} \in M_{i}$, for $i=1,2$. Let $L_{i}:=\left\langle p, x_{i}\right\rangle$. As $x_{i} \nsucc p$, $L_{i}$ meets $A$ in a point $p_{i}$. Clearly, $p_{1} \perp p_{2}$. Hence $x_{1} \perp x_{2}$. Consequently, $L^{\prime} \cup M_{1} \cup M_{2}$ is contained in a singular subspace of $\Delta$. However, this is impossible, as $\Delta$ has rank 3 . We avoid this contradiction only assuming that things are as described in the second part of (3).

Finally, suppose $L^{\prime} \notin \Gamma$. Then, for every plane $P \in \operatorname{Res}_{\Gamma}(L), p$ and the line $p^{\perp} \cap P$ are as in case (2) and the situation is as described in (4).

Lemma 4.9 Every quadrangle of $\mathcal{G}_{0}(\Gamma)$ splits in triangles.

Proof: Let $\left\{a_{0}, a_{1}, a_{2}, a_{3}\right\}$ be a quadrangle of $\mathcal{G}_{0}(\Gamma)$, with $a_{i} \sim a_{i+1}$ and $a_{i} \nsucc a_{i+2}$ for every $i=0,1,2,3$ (indices computed modulo 4). Let $L$ be the line of $\Gamma$ through $a_{2}$ and $a_{3}$. We have three cases to examine.

Case 1. $L \subset a_{0}^{\perp} \cap a_{1}^{\perp}$. Then both pairs $\left(a_{0}, L\right)$ and $\left(a_{1}, L\right)$ are as in case (2) of Lemma 4.8 . Hence $a_{0} \sim p \sim a_{1}$ for some point $p \in L$ and the quadrangle splits in the triangles $\left\{a_{0}, a_{1}, p\right\},\left\{a_{1}, a_{2}, p\right\},\left\{a_{0}, a_{3}, p\right\}$ and $\left\{a_{2}, a_{3}, p\right\}$ (the latter being contained in $L$ ).

Case 2. $a_{0}^{\perp} \cap L=a_{3}$ and $a_{1}^{\perp} \cap L=a_{2}$. Then we are in case (3) of Lemma 4.8. By Lemma 4.7, as $\Delta$ contains at least four planes on $L$, the geometry $\Gamma$ contains at least three planes on $L$. According to (3) of Lemma 4.8, for at least one of those planes, say $P$, both $a_{0}^{\sim} \cap P$ and $a_{1}^{\sim} \cap P$ are lines. These lines meet in a point $p$. Thus, we can split $\left\{a_{0}, a_{1}, a_{2}, a_{3}\right\}$ in four triangles, namely $\left\{a_{0}, a_{1}, p\right\},\left\{a_{1}, a_{2}, p\right\},\left\{a_{0}, a_{3}, p\right\}$ and $\left\{a_{2}, a_{3}, p\right\}$.

Case 3. $L$ is contained in $a_{i}^{\perp}$ but not in $a_{j}^{\perp}$, for $(i, j)=(0,1)$ or $(1,0)$. Suppose $L \subset a_{1}^{\perp}$ and $L \cap a_{0}^{\perp}=a_{3}$, to fix ideas. As $a_{1} \nsucc a_{3}$, the line $\left\langle a_{1}, a_{3}\right\rangle$ meets $A$ in a point $p$. As every line of $\Delta$ belongs to at least four planes, we can always take a plane $P$ of $\Delta$ on $p$ and $a_{0}$, distinct from the plane containing $\left\{a_{0}, a_{1}, a_{3}\right\}$ and such that $P \cap A=p_{P}\left(a_{1}\right) \cap A=p$. If $a_{1}^{\prime}$ is a point of $a_{2}^{\perp} \cap P$ different from $p$, then $a_{3} \not \perp a_{1}^{\prime}$, because $a_{3}^{\perp} \cap P=\left\langle a_{0}, p\right\rangle$. So, if we replace $a_{1}$ with $a_{1}^{\prime}$, we get a quadrangle as in Case 2 .

Lemma 4.10 Every bad triangle of $\mathcal{G}_{0}(\Gamma)$ splits in good triangles. 
Proof: Let $\left\{a_{1}, a_{2}, a_{3}\right\}$ be a bad triangle and $p$ the point $\left\langle a_{1}, a_{2}, a_{3}\right\rangle \cap A$ (compare Lemma 4.1). As every line of $\Delta$ belongs to at least four planes, we can take a plane $P \in \operatorname{Res}_{\Delta}(p)$ with the property that $P \cap A=P \cap\left\langle a_{1}, a_{2}, a_{3}\right\rangle=p$ and $p_{A}\left(a_{i}\right) \cap P=p$ for $i=1,2,3$. Given a line $L$ of $P$ not through $p$, let $P_{0}$ be a plane of $\Gamma$ on $L$, far from both $A$ and $\left\langle a_{1}, a_{2}, a_{3}\right\rangle$ in $\Delta$. Suppose that, for $i=1,2$ or 3 , a point $x$ of the line $a_{i}^{\perp} \cap P_{0}$ does not belong to $a_{i}^{\sim}$. Then the line $M:=\left\langle a_{i}, x\right\rangle$ meets $A$ in a point $y$. As both $p$ and $y$ belong to $A$, we have $p \perp y$. Hence $p \perp M$, as $a_{i} \perp p \perp y$. On the other hand, we also have $p \perp L$ and $L$ meets the line $a_{i}^{\perp} \cap P_{0}$ in a point $z$.

Suppose $z=x$. Then $M \subset p_{P}\left(a_{i}\right)$, hence $y \in p_{P}\left(a_{i}\right)$. Thus, $p_{P}\left(a_{i}\right)$ contains at least two points of $A$, namely $y$ and $p$. Consequently, $p_{P}\left(a_{i}\right)=p_{A}\left(a_{i}\right)$, hence $p_{A}\left(a_{i}\right) \cap P=M$, contrary to the assumption that $p_{P}\left(a_{i}\right) \cap A=p$. So, $z \neq x$. However, if so, $p$ is collinear with two distinct lines of $p_{P_{0}}\left(a_{i}\right)$, namely $M$ and the line $\left\langle a_{i}, z\right\rangle$. Hence $p \in p_{P_{0}}\left(a_{i}\right)$ (as $\Delta$ has rank 3). However this is impossible. Therefore, all points of $a_{i}^{\perp} \cap P_{0}$ are collinear with $a_{i}$ in $\Gamma$, namely: $p_{P_{0}}\left(a_{i}\right) \in \Gamma$.

For $\{i, j, k\}=\{1,2,3\}$, put $L_{i}:=\left\langle a_{j}, a_{k}\right\rangle$. Suppose that $p_{P_{0}}\left(L_{i}\right) \notin \Gamma$. Then $p_{P_{0}}\left(L_{i}\right) \cap A$ contains a point $x \in A$. We have $p \perp x$ and $x \notin L_{i}$. Moreover, $p \perp L_{i}$. Hence $p \in p_{P_{0}}\left(L_{i}\right)$, which is impossible. Therefore, $p_{P_{0}}\left(L_{i}\right) \in \Gamma$.

So far, we have proved that all planes $p_{P_{0}}\left(a_{i}\right)$ and $p_{P_{0}}\left(L_{i}\right)$ belong to $\Gamma$. Hence, with $b_{i}=L_{i}^{\perp} \cap P_{0}$, all triangles $\left\{b_{i}, a_{j}, a_{k}\right\}$ and $\left\{a_{i}, b_{j}, b_{k}\right\}$ are good. As the triangle $\left\{b_{1}, b_{2}, b_{3}\right\}$ is also good, we have decomposed $\left\{a_{1}, a_{2}, a_{3}\right\}$ in seven good triangles.

The conclusion of Theorem 1.2 follows from Lemmas 4.4, 4.9 and 4.10 via Proposition 2.1.

\section{Proof of Theorem 1.3}

Let $\Pi$ be the polar space associated to $\Delta$. The structure $\operatorname{Far}_{\Pi}(A)$ can be defined in the same way as $\operatorname{Far}_{\Delta}(A)$. However, as $\Pi$ is non-thick, $\operatorname{Far}_{\Pi}(A)$ is non-firm. So, $\operatorname{Far}_{\Pi}(A)$ is not a geometry in the sense of [13]. Actually, most of the theory of [13] (including Theorem 12.64, rephrased as Proposition 2.1 in this paper), also holds for residually connected but non-firm incidence structures, but one should rewrite too many parts of [13] to show this with full evidence. So, we shall argue differently.

Let $\Phi$ be the $\{n-1\}$-truncation of $\operatorname{Far}_{\Pi}(A)$. Then $\Phi$ is a geometry and the proof of Theorem 1.2 can be recycled to show that $\Phi$ is simply connected. Clearly, $\mathcal{G}_{0}(\Phi)=\mathcal{G}_{0}(\Gamma)$ where $\Gamma:=\operatorname{Far}_{\Delta}(A)$. All closed paths of $\mathcal{G}_{0}(\Gamma)$ that are good for $\Phi$ are also good for $\Gamma$. The simple connectedness of $\Gamma$ follows from this remark and the simple connectedness of $\Phi$, via Proposition 2.1.

\section{Proof of Theorem 1.4 in the generic case}

Troughout this section $\Gamma:=\operatorname{Far}_{\Delta}(F)$ where $\Delta$ is the building of type $D_{n}$ defined over a given field $K$ and $F$ is a $\{+,-\}$-flag as in Theorem 1.4, but we assume $n>5$ when $K=G F(2)$. 


\subsection{Notation and preliminaries}

As usual, we call the elements of $\Delta$ of type 0 and 1 points and lines respectively. The elements of $\Delta$ of type + or - are maximal singular subspaces of the polar space associated to $\Delta$. Accordingly, we call them maximal subspaces of $\Delta$. For an element $X$ of $\Delta$, let $\sigma(X)$ be the set of points (i.e. 0 -elements) of $\Delta$ incident to $X$. We take the liberty to write $X$ for $\sigma(X)$. So, given a set of points $S$ or a point $p$, we will write $X \subseteq S$ for $\sigma(X) \subseteq S$ and $p \in X$ for $p \in \sigma(X)$.

We denote by $U^{+}$and $U^{-}$the two maximal subspaces of $\Delta$ forming the flag $F$, with $U^{+}$ of type + and $U^{-}$of type - . We set $S:=U^{+} \cup U^{-}$and $S_{0}:=U^{+} \cap U^{-}$.

The geometry $\Gamma$ can be described as follows: The 0 -elements (points) of $\Gamma$ are the points of $\Delta$ that do not belong to $S$; for $i=1,2, \ldots, n-3$, the $i$-elements of $\Gamma$ are the $i$-elements of $\Delta$ that meet $S$ trivially; the elements of $\Gamma$ of type + and - (which we also call maximal subspaces of $\Gamma$ ) are the elements $U$ of $\Delta$ of type + or - such that $|U \cap S|=1$; the incidence relation is inherited from $\Delta$, but for stating that two maximal subspaces $X, Y$ are incident in $\Gamma$ only if $X \cap Y \cap S=\emptyset$.

We recall that two maximal subspaces $U, U^{\prime}$ of $\Delta$ have the same type if and only if $U \cap U^{\prime}$ has even codimension in any of $U$ or $U^{\prime}$. So, $\operatorname{dim}\left(U \cap U^{+}\right)+\operatorname{dim}\left(U \cap U^{-}\right)$is odd for every maximal subspace $U$. In particular, every maximal subspace of $\Delta$ meets at least one of $U^{+}$or $U^{-}$non-trivially and, if it meets $S_{0}$ non-trivially, then it has at least a line in common with at least one of $U^{+}$and $U^{-}$. So, the maximal subspaces of $\Delta$ that belong to $\Gamma$ are those which intersect $S_{0}$ trivially. Furthermore,

Lemma 6.1 For every element $X$ of $\Delta$ of type $i \leq n-3$ disjoint from both $U^{+}$and $U^{-}$, for $\{\varepsilon, \eta\}=\{+,-\}$ there exists a maximal subspace $U$ of $\Delta$ such that $U \supset X, U \cap U^{\varepsilon}=\emptyset$ and $\left|U \cap U^{\eta}\right|=1$.

Proof: As $X$ is far from $U^{\varepsilon}$, it is contained in a maximal subspace $U$ far from $U^{\varepsilon}$, namely such that $U \cap U^{\varepsilon}=\emptyset$. According to the above, $U \cap U^{\eta}$ is a point.

We now split our proof in two parts. We consider the case of $K \neq G F(2)$ first, obtaining the desired conclusion via Proposition 2.1 after a number of lemmas, as we have done in Sections 3 and 4. A different approach will be used in the case of $K=G F(2)$.

\subsection{The case of $K \neq G F(2)$}

In the sequel $K \neq G F(2)$. We denote by $\sim$ the collinearity relation of $\Gamma$, keeping the symbol $\perp$ for the collinearity relation of $\Delta$. Also, given a clique $X$ of the collinearity graph of $\Delta$, we denote by $\langle X\rangle$ the singular subspace spanned by $X$ in the polar space associated to $\Delta$. In particular, if $a, b$ are collinear points of $\Delta$, then $\langle a, b\rangle$ is the line of $\Delta$ through them.

Lemma 6.2 The graph $\mathcal{G}_{0}(\Gamma)$ has diameter 2 .

Proof: Given points $a, b$ of $\Gamma$, let $p$ be a point of $U^{+} \backslash S_{0}$ non-collinear with any of $a$ or $b$ (such a point exists as $K \neq G F(2)$ ). Let $U$ be a maximal subspace of $\Delta$ with $U \cap U^{+}=\{p\}$ 
and $U \cap U^{-}=\emptyset$ (Lemma 6.1). Then $U$ belongs to $\Gamma$. The point $p$, which is the unique point of $U \cap S$, belongs neither to $A:=a^{\perp} \cap U$ nor to $B:=b^{\perp} \cap U$. Put $V_{a}:=\langle a, A\rangle$ and $V_{b}:=\langle b, B\rangle$. As $A$ is a hyperplane of $V_{a}$ disjoint from $S$, the intersection $V_{a} \cap U^{+}$ is either a point or empty. Similarly, $V_{a} \cap U^{-}$is either a point or trivial. So $V_{a}$ belongs to $\Gamma$. Furthermore, one of $V_{a} \cap U^{+}$and $V_{a} \cap U^{-}$is a point and the other one is trivial. Consequently, exactly one of the lines of $V_{a}$ through $a$ meets $S$. Similarly, $V_{b}$ belongs to $\Gamma$ and exactly one of the lines of $V_{b}$ through $b$ meets $S$. Thus, and since $A \cap B$ is at least a line because $n \geq 4$, there is a point $d \in A \cap B$ joined with both $a$ and $b$ by lines avoiding $S$.

Lemma 6.3 Every closed path of $\mathcal{G}_{0}(\Gamma)$ splits in quadrangles and triangles.

Proof: By Lemma 6.2, every closed path of $\mathcal{G}_{0}(\Gamma)$ splits in pentagons, quadrangles or triangles. It remains to prove that every pentagon splits in quadrangles and triangles.

Let $a, b_{1}, b_{2}, c_{1}, c_{2}$ be points of $\Gamma$ forming a pentagon of $\mathcal{G}_{0}(\Gamma)$, namely $a \sim b_{i} \sim c_{i}$ for $i=1,2$ and $c_{1} \sim c_{2}$. Let $U$ be a maximal subspace on $\left\langle c_{1}, c_{2}\right\rangle$ disjoint from $U^{+}$and meeting $U^{-}$in exactly one point, say $p$. Put $A:=a^{\perp} \cap U$ and $V:=\langle a, A\rangle$. As $A$ is a hyperplane of both $U$ and $V$ and $U \cap U^{+}=\emptyset$, the intersection $V \cap U^{+}$is at most a point, whence $V \cap U^{-}$is at most a line. Thus, the set of points $x \in A$ such that $\langle x, a\rangle \cap S \neq \emptyset$ is contained in the join of a point and a line of $A$. Consequently, there are points $x \in A$ such that $\langle x, a\rangle \cap S=\emptyset$.

Lemma 6.4 Every quadrangle of $\mathcal{G}_{0}(\Gamma)$ splits in triangles.

Proof: Let $a_{1}, a_{2}, b_{1}, b_{2}$ be points forming a quadrangle, with $a_{i} \sim b_{j}$ for $i, j=1,2$. We may assume that neither $a_{1} \sim a_{2}$ nor $b_{1} \sim b_{2}$, otherwise there is nothing to prove. We have three cases to consider.

Case 1 Suppose that $a_{1} \not \perp \quad a_{2}$ and $b_{1} \not \perp b_{2}$. Let $U$ be a maximal subspace on $\left\langle a_{2}, b_{2}\right\rangle$ disjoint from $U^{+}$and meeting $U^{-}$in exactly one point, say $p$ (such a subspace exists by Lemma 6.1). Put $A:=a_{1}^{\perp} \cap U, B:=b_{1}^{\perp} \cap U, V:=\left\langle a_{1}, A\right\rangle$ and $W:=\left\langle b_{1}, B\right\rangle$. As $A$ is a hyperplane of $V$ and $V \cap U=A$, the maximal subspaces $V$ and $U$ have different type. So, $V \cap U^{+}$is a point, say $p_{V}$, and $V \cap U^{-}$is either a line or empty. Similarly, $W$ and $U$ have different type, $W \cap U^{+}$is a point $p_{W}$ and $W \cap U^{-}$is either a line or empty. Clearly, $W$ and $V$ have the same type. Suppose $V \cap U^{-}$is a line, say $L_{V}$. Then $L_{V}$ meets $S_{0}=U^{+} \cap U^{-}$ in a point, as $S_{0}$ is a hyperplane in $U^{-}$. It also meets $A$ in a point, since $A$ is a hyperplane of $V$. That point must be the same as $p$, since $p$ is the unique point of $U \cap U^{-}$. Hence $p \in L_{V}$. Furthermore, $p_{V} \in L_{V}$, as $L_{V} \cap S_{0} \subseteq U^{+} \cap V=p_{V}$. Similarly for $W$. Thus, the following are the cases that might occur, up to permuting $V$ and $W$ :

(i) Both $L_{V}=V \cap U^{-}$and $L_{W}=W \cap U^{-}$are lines through $p$ and both $p_{V}=L_{V} \cap S_{0}$ and $p_{W}=L_{W} \cap S_{0}$ are points different from $p$.

(ii) $W \cap U^{-}=\emptyset, p_{W}=W \cap U^{+}$is a point, $L_{V}=V \cap U^{-}$is a line through $p$ and $p_{V}=L_{V} \cap S_{0}$ is a point different from both $p$ and $p_{W}$.

(iii) $V \cap U^{-}=W \cap U^{-}=\emptyset$, whereas both $p_{V}=V \cap U^{+}$and $p_{W}=W \cap U^{-}$are points. 
Clearly, $a_{2} \in B$ and $b_{2} \in A$. Therefore, $b_{1} \notin V$ and $a_{1} \notin W$, as $a_{1} \not \perp a_{2}$ and $b_{1} \not \perp b_{2}$. Hence $V \neq W$, the subspace $X:=\left\langle a_{1}, b_{1}, A \cap B\right\rangle$ is maximal and $V \cap W=A \cap B$, of dimension $n-3$. Also, $X$ has type opposite to that of $V$ and $W$, as it meets each of them in an $(n-2)$-dimensional subspace, namely $\left\langle A \cap B, a_{1}\right\rangle$ and $\left\langle A \cap B, b_{1}\right\rangle$. Consequently, $X$ and $U$ have the same type and $X \cap U=A \cap B$.

Assume (i). The lines $L_{V}$ and $L_{W}$ are distinct and span a plane $\left\langle L_{V}, L_{W}\right\rangle$ of $\Delta$ contained in $U^{-}$. Suppose first $n>4$. Then a point $c \in A \cap B$ can always be chosen in such a way that each of the lines $\left\langle c, a_{1}\right\rangle$ and $\left\langle c, b_{1}\right\rangle$ misses $L_{V} \cup L_{W}$. Clearly, $c \neq p$. Furthermore, none of the lines $\left\langle a_{2}, c\right\rangle$ and $\left\langle b_{2}, c\right\rangle$ belongs to $A \cap B$. Hence each of them misses $S$, as $p \in A \cap B$ is the unique point of $U \cap S$. So, we have decomposed $\left\{a_{1}, b_{1}, a_{2}, b_{2}\right\}$ in four triangles, namely $\left\{a_{1}, b_{1}, c\right\},\left\{b_{1}, a_{2}, c\right\},\left\{a_{2}, b_{2}, c\right\}$ and $\left\{b_{2}, a_{1}, c\right\}$.

Suppose now that $n=4$. Then $\operatorname{dim}(X)=3$ and $A \cap B$ is a line. Furthermore, as $X$ has type opposite to $V$ and $V \cap U^{-}$is a line, $X \cap U^{-}$is either a plane or a point. If $X \cap U^{-}$is a plane, that plane meets the line $\left\langle a_{1}, b_{1}\right\rangle$ in a point, contrary to the fact that $\left\langle a_{1}, b_{1}\right\rangle$ belongs to $\Gamma$. Therefore $X \cap U^{-}$is a point, necessarily equal to $p$, as $p=$ $A \cap B \cap U^{-}$. Chosen a point $c \neq p$ in the line $A \cap B$, we get a splitting of $\left\{a_{1}, b_{1}, a_{2}, b_{2}\right\}$ in four triangles $\left\{a_{1}, b_{1}, c\right\},\left\{b_{1}, a_{2}, c\right\},\left\{a_{2}, b_{2}, c\right\}$ and $\left\{b_{2}, a_{1}, c\right\}$ of $\mathcal{G}_{0}(\Gamma)$, as in the case of $n>4$.

Suppose that (ii) occurs. As $L_{V} \cap A \cap B \subseteq V \cap U^{-} \cap A \cap B \subseteq V \cap U^{-} \cap W \subseteq U^{-} \cap W=\emptyset$, we have $L_{V} \cap A \cap B=\emptyset$. Consequently, $\left\langle a_{1}, x\right\rangle \cap S \neq \emptyset$ for at most one point $x \in A \cap B$. Similarly, $\left\langle b_{2}, y\right\rangle \cap S \neq \emptyset$ for at most one point $y \in A \cap B$ and $\left\langle b_{1}, z\right\rangle \cap S \neq \emptyset$ for at most one point $z \in A \cap B$. Furthermore, $\left\langle a_{2}, v\right\rangle \cap S=\emptyset$ for all points $v \in A \cap B$, as $p_{W} \notin B$. However, $|A \cap B| \geq 4$. (When $n=4$, recall that $K \neq G F(2)$.) Therefore, a point $c \in A \cap B$ can be chosen in such a way that each of the lines $\left\langle a_{i}, c\right\rangle$ and $\left\langle b_{i}, c\right\rangle$ misses $S$. The required decomposition is obtained.

If (iii) holds then the conclusion follows by an argument as above. Things are even easier now and there is no need to recall that $K \neq G F(2)$. We leave the details for the reader.

Case 2. Suppose that $a_{1} \perp a_{2}$ and $b_{1} \perp b_{2}$. Then each of the lines $\left\langle a_{1}, a_{2}\right\rangle$ and $\left\langle b_{1}, b_{2}\right\rangle$ meets $S$ in a point, as $a_{1} \nsucc a_{2}$ and $b_{1} \nsucc b_{2}$ by assumption. Hence the space $X:=\left\langle a_{1}, b_{1}, a_{2}, b_{2}\right\rangle$ meets $S$ in a point (if $X$ is a plane) or a line (when $\operatorname{dim}(X)=3$ ). If $X$ is a plane, then $c:=\left\langle a_{1}, b_{1}\right\rangle \cap\left\langle a_{2}, b_{2}\right\rangle$ is a point of $\Gamma$. Thus, we can decompose $\left\{a_{1}, b_{1}, a_{2}, b_{2}\right\}$ in $\left\{a_{1}, b_{1}, c\right\}$, $\left\{b_{1}, a_{2}, c\right\},\left\{a_{2}, b_{2}, c\right\}$ and $\left\{b_{2}, a_{1}, c\right\}$. On the other hand, when $\operatorname{dim}(X)=3$ we can always pick a point $c \in X$ such that each of the lines $\left\langle a_{1}, c\right\rangle,\left\langle b_{1}, c\right\rangle,\left\langle a_{2}, c\right\rangle$ and $\left\langle b_{2}, c\right\rangle$ misses $S$. Again, we obtain a decomposition of $\left\{a_{1}, b_{1}, a_{2}, b_{2}\right\}$ in triangles.

Case 3. The case where only one of the relations $a_{1} \perp a_{2}$ or $b_{1} \perp b_{2}$ holds remains to consider. Suppose $a_{1} \perp a_{2}$ but $b_{1} \not \perp b_{2}$, to fix ideas. Then $p:=\left\langle a_{1}, a_{2}\right\rangle \cap S$ is a point.

Suppose first $p \in S \backslash S_{0}$. Without loss, we may assume that $p \in U^{-}$. So, $p \notin U^{+}$as $p \notin S_{0}$. Pick a maximal subspace $U$ of $\Delta$ containing $\left\langle a_{1}, a_{2}, b_{2}\right\rangle$ and such that $U \cap U^{-}=p$. Then $U \cap U^{+}=\emptyset$, as $\left\langle p, p^{\perp} \cap U^{+}\right\rangle=U^{-}$. Given a point $c$ of $\left(b_{1}^{\perp} \cap U\right) \backslash\left\langle a_{1}, a_{2}\right\rangle$, we can decompose $\left\{a_{1}, b_{1}, a_{2}, b_{2}\right\}$ in $\left\{a_{1}, b_{2}, c\right\},\left\{a_{2}, b_{2}, c\right\}$ and $\left\{a_{1}, b_{1}, a_{2}, c\right\}$, which are triangles 
and quadrangles of $\mathcal{G}_{0}(\Gamma)$. (Recall that $U \cap S=p \in\left\langle a_{1}, a_{2}\right\rangle$.) However, the quadrangle $\left\{a_{1}, b_{1}, a_{2}, c\right\}$ is as in Case 2. So, it admits a decomposition in triangles.

Suppose that $p \in S_{0}$. By Lemma 6.1 applied to $\operatorname{Res}_{\Delta}(p)$, there exists a maximal subspace $U$ of $\Delta$ containing $\left\langle a_{1}, a_{2}, b_{2}\right\rangle$ and such that $U \cap U^{+}=p$ and $L:=U \cap U^{-}$is a line through $p$. Put $B:=U \cap b_{1}^{\perp}$ and $V:=\left\langle b_{1}, B\right\rangle$. The line $\left\langle a_{1}, a_{2}\right\rangle$ is contained in $B$ and $B \cap S$ is either $L$ or $p$. If $L \cap B=p$, then $V \cap U^{-}=p$ and $V \cap U^{+}$is a line $M$ through $p$ with $M \cap U^{-}=p$. Indeed $V$ and $U$ belong to opposite families, $U$ meets $U^{-}$in a line and $B$, which is a hyperplane of $V$, has only one point in common with $U^{-}$, namely $p$. In this case we can always pick a point $c \in B \backslash\left\langle a_{1}, a_{2}\right\rangle$ in such a way that $\left\langle c, b_{2}\right\rangle$ misses $L$ and $\left\langle c, b_{1}\right\rangle$ misses $M$. Thus, $\left\{a_{1}, b_{1}, a_{2}, b_{2}\right\}$ splits in the triangles $\left\{a_{1}, b_{1}, c\right\},\left\{b_{1}, a_{2}, c\right\}$, $\left\{a_{2}, b_{2}, c\right\}$ and $\left\{b_{2}, a_{1}, c\right\}$.

Finally, suppose $L \subset B$. Then $V \cap U^{-}$is a plane containing $L$, because $U$ and $V$ belong to different families. However, $V$ contains lines of $\Gamma$ and these lines miss the plane $V \cap U^{-}$. Therefore $\operatorname{dim}(V) \geq 4$. Furthermore, $V \cap S_{0}=V \cap U^{-} \cap U^{-}$. So, $V \cap S$ is equal to the plane $V \cap U^{-}$. As $\operatorname{dim}(V) \geq 4$ and $B$ is a hyperplane of $V$, the space $B$ contains a point $c$ that does not belong to the join of the planes $B \cap\left\langle V \cap U^{-}, b_{1}\right\rangle, B \cap\left\langle V \cap U^{-}, a_{1}\right\rangle$ and $B \cap\left\langle V \cap U^{-}, a_{2}\right\rangle$. (When $n=4$, recall that $K \neq G F(2)$.) For such a point $c$, each of the lines $\left\langle c, a_{1}\right\rangle,\left\langle c, a_{2}\right\rangle,\left\langle c, b_{1}\right\rangle$ and $\left\langle c, b_{2}\right\rangle$ misses $S$. Again, we have decomposed $\left\{a_{1}, b_{1}, a_{2}, b_{2}\right\}$ in triangles.

We say that a triangle $\{a, b, c\}$ of $\mathcal{G}_{0}(\Gamma)$ is degenerate if it is contained in a line of $\Gamma$. Clearly, all degenerate triangles are good.

Lemma 6.5 We have $\left|\langle a, b, c\rangle \cap S_{0}\right| \leq 1$ for every non-degenerate triangle $\{a, b, c\}$ of $\mathcal{G}_{0}(\Gamma)$, with equality holding if and only if $\{a, b, c\}$ is bad.

Proof: As the plane $\langle a, b, c\rangle$ contains lines that do not meet $S$, the intersection $\langle a, b, c\rangle \cap$ $S_{0}$ is at most a point. Let $U$ be a maximal singular subspace of $\Delta$ containing $\langle a, b, c\rangle$. Suppose that $\langle a, b, c\rangle \cap S_{0}=\emptyset$. Then $\langle a, b, c\rangle$ is disjoint from at least one of $U^{+}$and $U^{-}$. Consequently, it is contained in a maximal subspace of $\Delta$ that is disjoint from either $U^{+}$ or $U^{-}$and, therefore, belongs to $\Gamma$. In this case, $\{a, b, c\}$ is good. On the other hand, when $\langle a, b, c\rangle \cap S_{0} \neq \emptyset$ none of the singular subspaces of $\Delta$ containing $\langle a, b, c\rangle$ is disjoint from any of $U^{+}$or $U^{-}$. In that case $\{a, b, c\}$ is bad.

Lemma 6.6 Every bad triangle of $\mathcal{G}_{0}(\Gamma)$ admits a decomposition in good triangles.

Proof: Let $\{a, b, c\}$ be a bad triangle and $p:=\langle a, b, c\rangle \cap S$. By Lemma 6.5, $p \in S_{0}$. By Lemma 6.1 in $\operatorname{Res}_{\Delta}(p)$, we can choose a maximal subspace $U$ of $\Delta$ containing $\langle a, b, c\rangle$ and such that $L:=U \cap U^{-}$is a line through $p$ and $U \cap U^{+}=p$. As $\operatorname{dim}(U) \geq 3$ and $K \neq G F(2)$, we can also pick a point $d \in U \backslash\langle a, b, c\rangle$ such that none of the planes $\langle d, a, b\rangle$, $\langle d, b, c\rangle$ and $\langle d, c, a\rangle$ contains $p$ and each of the lines $\langle d, a\rangle,\langle d, b\rangle$ and $\langle d, c\rangle$ misses $L$. So, the triplets $\{d, a, b\},\{d, b, c\}$ and $\{d, c, a\}$ are triangles of $\mathcal{G}_{0}(\Gamma)$. They are good, by Lemma 6.5.

The simple connectedness of $\Gamma$ follows from Lemmas 6.3, 6.4 and 6.5 via Proposition 2.1. 


\subsection{The case of $K=G F(2)$ with $n>5$}

In this subsection, $K=G F(2)$ but $n>5$. The proof we will give for this case makes no use of Proposition 2.1. We will recognize certain configurations in $\Gamma$ that don't break when lifted to covers. Via them, we will be able to prove that the collinearity graph of the universal cover of $\Gamma$ has diameter 2 . The conclusion will follow.

Recall that, given a maximal subspace $U$ of $\Delta$, the mapping sending $X \in \operatorname{Res}_{\Delta}(U)$ to the set $X \cap U$ is injective. In view of this, in the sequel we will freely indentify a flag $\{X, U\}$ with the intersection $X \cap U$ whenever this minor abuse will be convenient.

Lemma 6.7 Given a line $L$ of $\Gamma$ and distinct points $a_{1}, a_{2} \in L$, for $i=1,2$ let $L_{i}$ be a line of $\Gamma$ incident to $a_{i}$ and distinct from $L$. Then one of the following occurs:

(1) Some of the maximal subspaces of $\Gamma$ contains all of $L, L_{1}$ and $L_{2}$.

(2) Modulo permuting $L_{1}$ and $L_{2}$, there exist a maximal subspace $U$ of $\Gamma$ containing $L \cup L_{1}$ and planes $A_{1}, A_{2}$ of $\Gamma$ such that $L_{i} \subset A_{i}$ for $i=1,2, A_{1} \subset U, U \cap A_{2}$ is a line and $A_{1}$ has at least one point in common with the line $U \cap A_{2}$.

(3) There exist a maximal subspace $U$ of $\Gamma$ containing $L$ and planes $A_{1}, A_{2}$ of $\Gamma$ such that $L_{i} \subset A_{i}, A_{i} \cap U$ is a line for $i=1,2$ and the lines $A_{1} \cap U$ and $A_{2} \cap U$ meet in a point.

Proof: Let $\mathcal{U}$ be the family of maximal subspaces of $\Delta$ containing $L$ and disjoint from $U^{+}$. Clearly, the elements of $\mathcal{U}$ belong to $\Gamma$. Suppose some $U \in \mathcal{U}$ contains $L_{1}$. If $U$ also contains $L_{2}$ then we are in case (1).

Assuming $L_{2} \not \subset U$, put $U^{\prime}:=\left\langle L_{2}^{\perp} \cap U, L_{2}\right\rangle$. As $U \cap U^{+}=\emptyset$ and $\left\{t(U), t\left(U^{\prime}\right)\right\}=\{+,-\}$, $U^{\prime} \cap U^{+}$is a point $p_{0}$ and $U^{\prime} \cap U^{-}$is either empty or a line through $p_{0}$. However, $\operatorname{dim}\left(U^{\prime}\right)=$ $n-1 \geq 5$. Hence $U^{\prime}$ contains at least $m:=2^{n-2}-4 \geq 12$ planes $X_{1}, X_{2}, \ldots, X_{m}$ on $L_{2}$ with no points in $S=U^{+} \cup U^{-}$. As $U \cap U^{\prime}$ is a hyperplane of $U^{\prime}$, each of those planes meets $U \cap U^{\prime}$ in a line. For $i=1,2, \ldots, m$, put $M_{i}:=X_{i} \cap U \cap U^{\prime}=X_{i} \cap U$. Let $V$ be a maximal subspace of $\Gamma$ incident with $L_{1}$ and forming a $\{+,-\}$-flag with $U$. The dual of $\operatorname{Res}_{\Gamma}(U)$ is an affine geometry and we may regard $U \cap V$ as a point of that affine geometry. Therefore, for $i=1,2, \ldots, m$ either $U \cap V \supset M_{i}$ or $V \cap U \cap M_{i}$ is a point. Suppose first that $U \cap V$ contains one of the lines $M_{i}$, say $U \cap V \supset M_{1}$ to fix ideas. Then both $L_{1}$ and $M_{1}$ belong to $\operatorname{Res}_{\Gamma}(U, V)$. As $\operatorname{Res}_{\Gamma}(U, V)$ is a projective geometry, any point of $M_{1}$ is coplanar with $L_{1}$ and we have case (2) (with $A_{2}=X_{1}$ ).

Assume now that none of the lines $M_{i}$ is contained in $U \cap V$. Then $p_{i}:=M_{i} \cap U \cap V$ is a point. Suppose first $p_{i}=p_{j}$ for two disctinct indices $i, j$. Then $p_{i}=p_{j}=a_{2}$, as $M_{i} \neq M_{j}$ and them both are incident to $a_{2}$. Hence $a_{2} \in U \cap V . \operatorname{In}_{\operatorname{Res}_{\Gamma}}(U, V)$ we find a plane containing $L_{1}$ and $a_{2}\left(=p_{i}=p j\right)$ and we have case (2) with any of $X_{i}$ or $X_{j}$ taken as $A_{2}$.

Suppose the points $p_{1}, p_{2}, \ldots, p_{m}$ are pairwise different. Denoted by $\mathcal{A}$ the dual of $\operatorname{Res}_{\Gamma}(U)$, we have $\mathcal{A} \cong A G(n-1,2), U \cap V$ is a point of $\mathcal{A}$ and $p_{1}, p_{2}, \ldots, p_{m}$ appear as hyperplanes of $\mathcal{A}$ on the point $U \cap V$. The line $L_{1}$, regarded as an element of $\mathcal{A}$, is a subspace of codimension 2 . As the hyperplanes $p_{1}, p_{2}, \ldots, p_{m}$ are mutually non-parallel, the space $L_{1}$ is non-parallel with at least $m-3 \geq 9$ of them. Therefore, some $p_{i}$ meets $L_{1}$ in a $(n-4)$-space of $\mathcal{A}$. Namely, the point $p_{i}$ and the line $L_{1}$ are coplanar in $\operatorname{Res}_{\Gamma}(U)$, as in case (2). 
If some members of $\mathcal{U}$ contain $L_{2}$, then we obtain the same conclusion as above, but permuting $L_{1}$ and $L_{2}$. So, suppose that no member of $\mathcal{U}$ contains any of $L_{1}$ or $L_{2}$. Consequently, none of $L_{1}$ or $L_{2}$ is coplanar with $L$ in $\Gamma$. Given $U \in \mathcal{U}$, for $i=1,2$ let $V_{i}$ be the maximal subspace of $\Delta$ containing $L_{i}$ and forming a $\{+,-\}$-flag with $U$. (The element $V_{i}$ is uniquely determined inside $\operatorname{Res}_{\Delta}\left(a_{i}\right)$.) The subspace $V_{i}$ meets $S=U^{+} \cup U^{-}$in either a point or a line. Consequently, for $i=1,2$ there exist at least $m=2^{n-2}-4$ planes $X_{i, 1}, X_{i, 2}, \ldots, X_{i, m}$ of $\Gamma$ contained in $V_{i}$, containing $L_{i}$ and meeting $U$ in a line. For $j=1,2, \ldots, m$, put $M_{i, j}:=X_{i, j} \cap U$. Clearly, $M_{i, j} \neq M_{i, h}$ if $j \neq h$, as $X_{i, j} \cap X_{i, h}=L_{i} \nsubseteq U$. Furthermore, $M_{i, j} \neq L$, as $L_{i}$ and $L$ are non-coplanar in $\Gamma$. Denoted by $\mathcal{A}_{i}$ the dual of $\operatorname{Res}_{\Gamma}\left(a_{i}, U\right)$, we have $\mathcal{A}_{i} \cong A G(n-2,2)$ and $M_{i, 1}, M_{i, 2}, \ldots, M_{i, m}$ are hyperplanes of $\mathcal{A}_{i}$ containing the point $V_{i} \cap U$ of $\mathcal{A}_{i}$. At most one of those hyperplanes is parallel to $L$ in $\mathcal{A}_{i}$. To fix ideas, suppose $M_{i, j}$ is non-parallel to $L$ in $\mathcal{A}_{i}$ for $j=1,2, \ldots, m-1$ and let $Y_{i, j}$ be the meet of $L$ and $M_{i, j}$ in $\mathcal{A}_{i}$. So, $Y_{i, j}$ is the plane of $\Gamma$ containing $M_{i, j} \cup L$.

If $Y_{i, j}=Y_{i, h}$ for $j \neq h$ then $Y_{i, j}$ has at least two lines in common with $U \cap V_{i}$, namely $M_{i, j}$ and $M_{i, h}$. Therefore $Y_{i, j} \subset U \cap V_{i}$ and, consequently, $L \subset U \cap V_{i}$. Furthermore, $\left\{M_{i, j}, M_{i, h}, L\right\}$ is the triple of lines incident to $Y_{i, j}$ and $a_{i}$. Conversely, suppose $L \subset U \cap V_{i}$ and for every $j=1,2, \ldots, m-1$, let $Z_{j}$ be the 3-space of $\operatorname{Res}_{\Delta}\left(V_{i}\right)$ containing $Y_{i, j}$ and $L_{i}$. As $Z_{j}$ contains a plane of $\Gamma$, namely $Y_{i, j}, Z_{j}$ has at most one point in $S$ (recall that $V_{i} \cap S$ is either a point or a line). On the other hand, $Z_{j}$ contains the plane of $\Delta$ spanned by $L$ and $L_{i}$. By assumption, that plane does not belong to $\Gamma$, hence it contains a point of $S$. Thus, $Z_{j}$ meets $S$ in one point, which belongs to the plane spanned by $L_{i}$ and $L$. Consequently, $Z_{j}$ contains two planes of $\Gamma$ on $L_{i}$. The plane $Y_{i, j}$ is one of those two. The other plane is $Y_{i, f_{i}(j)}$ for a uniquely determined index $f_{i}(j) \neq j$. Thus, one of the following holds:

(*) $L \nsubseteq V_{i}$ and the planes $Y_{i, 1}, Y_{i, 2}, \ldots, Y_{i, m-1}$ are pairwise distinct;

(**) $L \subset V_{i}$, and $Y_{i, j}=Y_{i, f_{i}(j)}$ for an involutory fixed-point-free permutation $f_{i}$ of $\{1,2, \ldots, m\}$.

(Note that in case (**) none of the lines $M_{i, j}$ is parallel to $L$ in $\mathcal{A}_{i}$, as $m-1$ is odd.) Assume case (*), with $i=1$ to fix ideas. Suppose that $Y_{1, j} \neq Y_{2, h}$ for any $j=1,2, \ldots, m-1$ and $h=1,2, \ldots$. Then we get at least $m-1+m / 2 \geq 2^{n-2}-5+2^{n-3}-2=2^{n-2}+2^{n-3}-7$ planes on $L$ in $\operatorname{Res}_{\Gamma}(U)$. But $\operatorname{Res}_{\Gamma}(U)$ contains exactly $2^{n-2}-2$ planes on $L$. Therefore, $2^{n-3} \leq 7$, which forces $n \leq 5$, contrary to the assumption $n>5$. So, $Y_{1, j}=Y_{2, h}$ for suitable indices $j$ and $h$, whence the lines $M_{1, j}$ and $M_{2, h}$ are coplanar and, therefore, they meet in a point. We have case (3) with $A_{1}=X_{1, j}$ and $A_{2}=X_{2, h}$.

Finally, suppose we have $(* *)$ for both $i=1$ and $i=2$. We may assume to have chosen indices in such a way that $f_{1}(i)=f_{2}(i)=i+m / 2$ for $i=1,2, \ldots, m / 2$. So, $Y_{1,1}, \ldots, Y_{1, m / 2}$ and $Y_{2,1}, \ldots, Y_{2, m / 2}$ are the planes to consider. Note that $L \subset U \cap V_{1} \cap V_{2}$, as $L \subset U \cap V_{1}$ and $L \subset U \cap V_{2}$. For $i=1,2$, there are exactly $2^{n-3}-1$ planes on $L$ in $\operatorname{Res}_{\Delta}\left(U \cap V_{i}\right)$. As $m / 2 \geq 2^{n-3}-2$, at most one of those planes is missing in the family $\mathcal{Y}_{i}:=\left\{Y_{i, j}\right\}_{j=1}^{m / 2}$. However, as $n>5$ by assumption, there are exactly $2^{n-4}-1 \geq 3$ planes of $\Delta$ between $U \cap V_{1} \cap V_{2}$ and $L$. By the above, at least $2^{n-4}-2$ of them come from the family $\mathcal{Y}_{i}$. As $2 \cdot\left(2^{n-4}-2\right)>2^{n-4}-1$, some of those planes belongs to $\mathcal{Y}_{1} \cap \mathcal{Y}_{2}$. Suppose 
$Y_{1,1}=Y_{2,1}$ is such a plane. In $\operatorname{Res}_{\Gamma}\left(Y_{1,1}\right)$ we see that the lines $M_{1,1}$ and $M_{2,1}$ meet in a point. Thus, we have case (3) with $A_{1}=X_{1,1}$ and $A_{2}=X_{2,1}$.

Let $\varphi: \widetilde{\Gamma} \rightarrow \Gamma$ be the universal covering of $\Gamma$.

Lemma 6.8 The collinearity graph $\mathcal{G}_{0}(\widetilde{\Gamma})$ of $\widetilde{\Gamma}$ has diameter 2 .

Proof: Assuming that $\widetilde{\Gamma}$ contains a pair of points at distance 3, we will show that those points have distance $\leq 2$. That contradiction will establish our Lemma.

Given two points $\tilde{b}_{1}, \tilde{b}_{2}$ of $\widetilde{\Gamma}$ at distance 3 , let $\left(\tilde{b}_{1}, \tilde{a}_{1}, \tilde{a}_{2}, \tilde{b}_{2}\right)$ be a path of $\mathcal{G}_{0}(\widetilde{\Gamma})$ from $\tilde{b}_{1}$ to $\tilde{b}_{2}$. For $i=1,2$ let $\widetilde{L}_{i}$ be the line through $\tilde{b}_{i}$ and $\tilde{a}_{i}$ and $\widetilde{L}$ the line through $\tilde{a}_{1}$ and $\tilde{a}_{2}$. As $\varphi$ is a covering, the line $L:=\varphi(\widetilde{L})$ is different from $L_{i}:=\varphi\left(\widetilde{L}_{i}\right)$ and the points $a_{1}:=\varphi\left(\tilde{a}_{1}\right)$ and $a_{2}:=\varphi\left(\tilde{a}_{2}\right)$ are different. Thus, $a_{1}, a_{2}, L, L_{1}$ and $L_{2}$ are as in the hypotheses of Lemma 6.7. Suppose case (1) of that lemma occurs and let $U$ be a maximal subspace of $\Gamma$ incident to $L, L_{1}$ and $L_{2}$. As $\varphi$ is a covering, $U$ lifts via $\varphi$ to a uniquely determined element $\widetilde{U}$ of $\widetilde{\Gamma}$ on $\widetilde{L}$. As $\varphi$ isomorphically maps $\operatorname{Res}\left(\widetilde{L}_{i}\right)$ onto $\operatorname{Res}_{\Gamma}\left(L_{i}\right)$ and $L_{i} \subset U$, the line $\widetilde{L}_{i}$ is incident to $\widetilde{U}$. Thus, both $\tilde{b}_{1}$ and $\tilde{b}_{2}$ are incident to $\widetilde{U}$. However, $\operatorname{Res}(\widetilde{U})$ is isomorphic to the dual of $A G(n-1,2)$ and the collinearity graph of a dual affine geometry has diameter 2 , contrary to the hypothesis that $\tilde{b}_{1}$ and $\tilde{b}_{2}$ have distance 3 .

So, case (1) of Lemma 6.7 cannot occur. Assume that (2) occurs, namely there exist a maximal subspace $U$ of $\Gamma$ containing $L \cup L_{1}$ and planes $A_{1}, A_{2}$ of $\Gamma$ such that $L_{i} \subset A_{i}$ for $i=1,2, A_{1} \subset U, M:=U \cap A_{2}$ is a line and $A_{1} \cap M$ contains a point $p$. Let $\widetilde{U}, \widetilde{A}_{1}, \widetilde{A}_{2}$ and $\widetilde{M}$ be the preimages of $U, A_{1}, A_{2}$ and $M$ in $\operatorname{Res}(\widetilde{L}), \operatorname{Res}\left(\widetilde{L}_{1}\right), \operatorname{Res}\left(\widetilde{L}_{2}\right)$ and $\operatorname{Res}\left(\tilde{a}_{2}\right)$. As $\varphi$ induces an isomorphism from $\operatorname{Res}\left(\tilde{a}_{i}\right)$ to $\operatorname{Res}_{\Gamma}\left(a_{i}\right)$, both $\widetilde{A}_{1}$ and $\widetilde{M}$ are incident to $\widetilde{U}$ and $\widetilde{M}$ is incident to $\widetilde{A}_{2}$. Again, $\varphi$ induces an isomorphism from $\operatorname{Res}(\widetilde{U})$ to $\operatorname{Res}_{\Gamma}(U)$. Hence the point $p \in A_{1} \cap M$ lifts via $\varphi$ to a point $\tilde{p}$ incident to $\widetilde{A}_{1}$ and $\widetilde{M}$, whence to $\widetilde{A}_{2}$ too, as $\widetilde{M}$ is incident to $\widetilde{A}_{2}$. As we can see in $\operatorname{Res}\left(\widetilde{A}_{1}\right)$ and $\operatorname{Res}\left(\widetilde{A}_{2}\right)$, the point $\tilde{p}$ is collinear with both $\tilde{b}_{1}$ and $\tilde{b}_{2}$, contrary to the assumption that $\tilde{b}_{1}$ and $\tilde{b}_{2}$ have distance 3 .

So, case (2) of Lemma 6.7 is also impossible. Case (3) remains to consider: there exist a maximal subspace $U$ containing $L$ and planes $A_{1}, A_{2}$ such that $L_{i} \subset A_{i}, M_{i}:=A_{i} \cap U$ is a line and the lines $M_{1}$ and $M_{2}$ meet in a point $p$. The elements $U, A_{i}$ and $M_{i}$ lift to a maximal element $\widetilde{U}$ incident to $\widetilde{L}$, a plane $\widetilde{A}_{i}$ incident to $\widetilde{L}_{i}$ and a line $\widetilde{M}_{i}$ incident to $\tilde{a}_{i}$ and, as $\varphi$ induces on $\operatorname{Res}\left(\tilde{a}_{i}\right)$ an isomorphism to $\operatorname{Res}_{\Gamma}\left(a_{i}\right)$, the line $\widetilde{M}_{i}$ is incident to both $\widetilde{A}_{i}$ and $\widetilde{U}$. Similarly, the point $p$ lifts to a unique point $\tilde{p}$ of $\operatorname{Res}(\widetilde{U})$ incident to both $\widetilde{M}_{1}$ and $\widetilde{M}_{2}$. For $i=1,2$ the points $\tilde{p}$ and $\tilde{b}_{i}$ are collinear in $\operatorname{Res}\left(\widetilde{A}_{i}\right)$. Once again, we have reached a contradiction.

We can now finish the proof. By way of contradiction, suppose $\varphi$ is not an isomorphism. Then $\varphi\left(\tilde{a}_{1}\right)=\varphi\left(\tilde{a}_{2}\right)$ for distinct points $\tilde{a}_{1}$ and $\tilde{a}_{2}$ of $\widetilde{\Gamma}$. The points $\tilde{a}_{1}$ and $\tilde{a}_{2}$ are distinct and non-collinear, as $\varphi$ is a covering. However, by Lemma 6.8, there is a point $\tilde{c}$ collinear with both $\tilde{a}_{1}$ and $\tilde{a}_{2}$. For $i=1,2$ let $\widetilde{L}_{i}$ be the line through $\tilde{a}_{i}$ and $\tilde{c}$. Clearly, $\widetilde{L}_{1} \neq \widetilde{L}_{2}$. Hence the lines $L_{1}:=\varphi\left(\widetilde{L}_{1}\right)$ and $L_{2}:=\varphi\left(\widetilde{L}_{2}\right)$ are different, as $\varphi$ induces an injective mapping on $\operatorname{Res}(\tilde{c})$. Thus, we have two distinct lines $L_{1}, L_{2}$ of $\Gamma$ with two distinct points 
in common, namely $c:=\varphi(\tilde{c})$ and $a:=\varphi\left(\tilde{a}_{1}\right)=\varphi\left(\tilde{a}_{2}\right)$. This is impossible. Therefore $\varphi$ is an isomorphism. The theorem is proved in this case too.

Remark 6.9 The hypothesis $K=G F(2)$ is not strictly necessary for the above, but we need $K$ to be finite, in view of some arithmetical arguments used in the proof of Lemma 6.7. On the other hand, $K$ was allowed to be infinite in Section 6.2.

\section{Proof of Theorem 1.5}

The basic idea of this proof resembles that used for Theorem 1.4 in the case of $K=G F(2)$ : we shall prove that a certain subgraph of the incidence graph of the universal cover has a very small diameter and we will exploit that fact to show that the covering is an isomorphism.

Firstly, we state some terminology. Given $\Delta$ as in Theorem 1.5, we call the elements of $\Delta$ of type 0 and 1 points and lines, respectively, writing $x \perp y$ for two points $x, y$ when they are collinear. With points and lines chosen as above, $\mathcal{G}_{0}(\Delta)$ is the collinearity graph of $\Delta$. It is well known that any two points of $\Delta$ are incident with at least one common 4-element [13, Section 7.6.1]. Hence $\mathcal{G}_{0}(\Delta)$ has diameter 2 .

The elements of $\Delta$ may be regarded as distinguished subspaces of the point-line system of $\Delta$. Accordingly, given an element $A$ of $\Delta$ and a point $x$ or a subset $X$ of the point-set of $\Delta$, we write $x \in A$ if $x$ is incident to $A, X \subseteq A$ if all points of $X$ are incident to $A$, and so on. The claims gathered in the next lemma are straightforward.

Lemma 7.1 All the following hold for a point $p$ of $\Delta$ :

(1) A point $x$ of $\Delta$ is non-collinear with $p$ if and only if there exists a unique 4-element of $\Delta$ incident to both $x$ and $p$.

(2) For $1 \leq i \leq 4$, an $i$-element $X$ of $\Delta$ is far from $p$ if and only if $X \cap p^{\perp}=\emptyset$.

(3) A 5-element $X$ of $\Delta$ is far from $p$ if and only if $\left|X \cap p^{\perp}\right|=1$.

Given a point $p$ of $\Delta$, put $\Gamma:=\operatorname{Far}_{\Delta}(p)$. The next Lemma easily follows from Lemma 7.1.

Lemma 7.2 All the following hold:

(1) We have $\operatorname{Res}_{\Gamma}(X)=\operatorname{Res}_{\Delta}(X)$ for every 4-element $X$ of $\Gamma$.

(2) Given a point $x$ of $\Gamma$, let $A_{x}$ be the unique 4-element of $\Delta$ incident to both $x$ and $p$ (compare Lemma 7.1(1)). Then $\operatorname{Res}_{\Gamma}(x)$ is the subgeometry of $\operatorname{Res}_{\Delta}(x)$ far from $A_{x}$.

(3) Given a 5-element $X$, let $p_{X}$ be the unique point of $X$ collinear with $p$ (compare Lemma 7.1(3)). Then $\operatorname{Res}_{\Gamma}(X)$ is the dual affine space obtained from $\operatorname{Res}_{\Delta}(X)$ by removing $p_{X}$ and all elements incident to it.

Lemma 7.3 Let $\widetilde{\Gamma}$ be a geometry over the set of types $\{0,1,2,3,4,5\}$ where the residues of the elements of type 0,4 and 5 are as in $\Gamma$. Then for any two 4-elements of $\widetilde{\Gamma}$ there exists a 0-element incident to them both.

Proof: We shall prove that, given three 4-elements $A, B, C$ of $\widetilde{\Gamma}$, if there exist a 0 -element $a$ incident to both $A$ and $B$ and a 3-element $S$ incident to both $B$ and $C$, then there exists 
a 0 -element that is incident to both $A$ and $C$. Once we have proved this, the conclusion follows by considering for every 4-element $X$ a path of type $(4,3,4, \ldots, 3,4)$ from $B$ to $X$ in the incidence graph of $\widetilde{\Gamma}$.

Let $A, B, C, a, S$ be as above. By assumption, $\operatorname{Res}(B)$ is a building of type $D_{5}$. Hence there exists a 5-element $U \in \operatorname{Res}(B)$ incident to both $a$ and $S$. On the other hand, $\operatorname{Res}(a)$ is isomorphic to the subgeometry of a building $\Delta_{a}$ of type $D_{5}$ far from a given point $A_{a}$ of $\Delta_{a}$. Denoted by $\sim$ the collinearity relation of $\Delta_{a}$ and regarded $U$ as a maximal singular subspace of the polar space $\Pi_{a}$ associated to $\Delta_{a}$, suppose first that $A^{\sim} \cap U \subseteq A_{a}^{\sim}$. Then the 3-space $X:=A^{\sim} \cap U=A_{a}^{\sim} \cap U$ is contained in three maximal singular subspaces of $\Pi_{a}$, namely $U,\langle X, A\rangle$ and $\left\langle X, A_{a}\right\rangle$. This is impossible, as every singular 3-space of $\Pi_{a}$ is contained in exactly two maximal singular subspaces. Hence $A^{\sim} \cap U \nsubseteq A_{a}^{\sim}$.

In $\Pi_{a}$, given a point $A^{\prime} \in A^{\sim} \cap U \backslash A_{a}^{\sim}$, let $S^{\prime}$ be the line through $A$ and $A^{\prime}$. In the residue of $A^{\prime}$ in $\Pi_{a}$ we find a maximal singular subspace $U^{\prime}$ incident to $S^{\prime}$ and intersecting $U$ in a 3 -space. Clearly, $U^{\prime}$ is a 1-element of $\widetilde{\Gamma}$. We now turn to $\operatorname{Res}(U)$, where both $U^{\prime}$ and $C$ live. The dual of $\operatorname{Res}(U)$ is an affine geometry and, in that affine geometry, $C$ and $U^{\prime}$ are a point and a 3 -space respectively. Hence there exists a hyperplane $h$ of that affine geometry incident to both $C$ and $U^{\prime}$. The element $h$ is in fact a 0 -element of $\widetilde{\Gamma}$, it is incident to $C$ and, as it is incident to $U^{\prime}$, it is incident to $A$, too. The claim is proved.

Now we can finish the proof of Theorem 1.5. Let $\varphi: \widetilde{\Gamma} \rightarrow \Gamma$ be a covering and suppose that $\varphi(A)=\varphi(B)$ for two 4-elements $A, B$ of $\widetilde{\Gamma}$. By Lemma 7.3, there exists a 0-element $a$ incident to both $A$ and $B$. As $\varphi$ is a covering, its restriction to $\operatorname{Res}(a)$ is injective. Hence $A=B$. So, $\varphi$ is injective on the set of 4-elements. This forces $\varphi$ to be an isomorphism.

\section{Proof of Corollary 1.7}

A geometry $\Gamma$ of rank $n \geq 3$ is said to be residually simply connected if it is simply connected and, when $n>3$, all residues of $\Gamma$ of rank at least 3 are simply connected. Clearly, a residually simply connected geometry is also 2 -simply connected. So, we only need to prove the following:

Lemma 8.1 All far-away geometries considered in Theorems 1.1, 1.3 and 1.5 are residually simply connected. The geometries of Theorem 1.2 are residually connected provided that $\Delta$ is neither $\mathcal{S}(2 n-1,2)$ nor $\mathcal{H}(2 n-1,4)$. The geometries of Theorem 1.4 are residually connected provided that $\Delta \neq D_{n}(2)$.

Proof: The main step of the proof is to show that the residues of the flags of the considered far-away geometry $\Gamma$ are either far-away geometries of the same kind as $\Gamma$, but of lesser rank, or projective or affine geometries, or affine polar spaces (Section 1.1, Example 1.3), or affinedual-affine geometries of rank 3 (Section 1.1, Example 1.2), or direct sums of geometries as above. Once this is proved, the conclusion follows. Indeed projective geometries, affine geometries, affine polar spaces, affine-dual-affine geometries and direct sums are simply connected whereas, when smaller far-away geometries of the same kind as $\Gamma$ are involved, we can argue by induction. 
We are not going to examine all cases one by one. We shall only consider one of them, just to show how to do, leaving the remaining cases for the reader.

Given $\Delta$ and $F$ as in Theorem 1.4, let $p$ be a point of $\Gamma=\operatorname{Far}_{\Delta}(F)$ and $U^{+}, U^{-}$the two maximal subspaces of $\Delta$ forming $F$. As $p$ belongs to $\Gamma, p \notin U^{+} \cup U^{-}$. Put $\Delta_{p}:=\operatorname{Res}_{\Delta}(p)$ and $U_{p}^{\varepsilon}:=\left\langle p, p^{\perp} \cap U^{\varepsilon}\right\rangle$ for $\varepsilon=+,-$. Then $F_{p}:=\left\{U_{p}^{+}, U_{p}^{-}\right\}$is a flag of $\Delta_{p}$ of type $\{+,-\}$ and $\operatorname{Res}_{\Gamma}(p) \cong \operatorname{Far}_{\Delta_{p}}\left(F_{p}\right)$.

\section{Proof of claims (1) and (2) of Theorem 1.6}

Throughout this section $\Gamma_{1, n}:=\operatorname{Far}_{\Delta}(A)$ for $\Delta=\mathcal{S}(2 n-1,2)$ and $\Gamma_{2, n}:=\operatorname{Far}_{\Delta}(A)$ for $\Delta=\mathcal{H}(2 n-1,4)$, with $A$ an $(n-1)$-element of $\Delta$ in both cases and $n \geq 3$.

\subsection{Preliminaries}

It is known [16, Lemmas 6.1 and 6.5] that the dual of $\Gamma_{1, n}$ is isomorphic to the expansion $\operatorname{Exp}\left(\varepsilon_{1, n}\right)$ of a $G F(2)$-embedding $\varepsilon_{1, n}: P G(n-1,2) \rightarrow V\left(\left(\begin{array}{c}n+1 \\ 2\end{array}\right), 2\right)$, called the tensor embedding, and the dual of $\Gamma_{2, n}$ is isomorphic to the expansion of a $G F(2)$-embedding $\varepsilon_{2, n}: P G(n-1,4) \rightarrow V\left(n^{2}, 2\right)$, called the twisted tensor embedding. Referring the reader to [16, Section 6] for more details, we recall the definitions of $\varepsilon_{1, n}$ and $\varepsilon_{2, n}$.

Definition of $\varepsilon_{1, n} \quad$ For a nonzero vector $v \in V=V(n, 2), \varepsilon_{1, n}$ sends the point $\langle v\rangle$ of $P G(n-1,2)$ to the vector $v \otimes v$. These vectors span an $\left(\begin{array}{c}n+1 \\ 2\end{array}\right)$-dimensional subspace $V_{1}$ of $V \otimes V$. In fact, if $\left\{e_{i}\right\}_{i=1}^{n}$ is a basis of $V$, then

$$
\left\{e_{i} \otimes e_{i}\right\}_{i=1}^{n} \cup\left\{e_{i} \otimes e_{j}+e_{j} \otimes e_{i}\right\}_{i<j}
$$

is a basis of $V_{1}$. The additive group of $V_{1}$ can also be recovered inside the stabilizer of $A$ in $S_{2 n}(2)=\operatorname{Aut}(\Delta)$ as the unipotent radical $U$ of that parabolic subgroup. The group $U$ acts regularly on the set of 3-elements of $\Gamma_{1, n}$. The isomorphism $\Gamma_{1, n} \cong \operatorname{Exp}\left(\varepsilon_{1, n}\right)$ is implicit in that fact.

Definition of $\varepsilon_{2, n}$ Given a basis $\left\{e_{i}\right\}_{i=1}^{n}$ of $V=V(n, 4)$, for every non-zero vector $v=$ $\sum_{i=1}^{n} t_{i} e_{i}$ of $V$ we put $\bar{v}:=\sum_{i=1}^{n} t_{i}^{2} e_{i}$ and $\varepsilon_{2, n}(\langle v\rangle):=v \otimes \bar{v}$. As $(t v) \otimes \overline{(t v)}=t^{3} v \otimes \bar{v}=v \otimes \bar{v}$ for every non-zero scalar $t, \varepsilon_{2, n}$ is indeed a mapping from the set of points of $P G(n-1,4)$ to the set of non-zero vectors of $V \otimes V$. Regarded the latter as a $2 n^{2}$-dimensional $G F(2)$ vector space, the vectors $v \otimes \bar{v}$ span an $n^{2}$-dimensional subspace $V_{2}$ in it. Explicitly, given $\omega \in G F(4) \backslash G F(2)$, the vectors $e_{i, j}:=e_{i} \otimes e_{j}$ and $f_{i, j}:=\omega e_{i} \otimes e_{j}$ form a $G F(2)$-basis of $V \otimes V$ and the set

$$
\left\{e_{i, i}\right\}_{i=1}^{n} \cup\left\{e_{i, j}+e_{j, i}\right\}_{i<j} \cup\left\{e_{i, j}+f_{i, j}+f_{j, i}\right\}_{i<j}
$$

is a basis of $V_{2}$. The additive group of $V_{2}$ is isomorphic to the unipotent radical $U$ of the stabilizer of $A$ in $U_{2 n}(2)=\operatorname{Aut}(\Delta)$. The group $U$ acts regularly on the set of 3-elements of $\Gamma_{2, n}$ and this fact implies that $\Gamma_{2, n} \cong \operatorname{Exp}\left(\varepsilon_{2, n}\right)$. 
By Proposition 2.5 we immediately obtain the following, which in principle solves the problem of determining the universal 2 -covers of $\Gamma_{1, n}$ and $\Gamma_{2, n}$ :

Lemma 9.1 For $i=1,2$, the universal 2-cover of $\Gamma_{i, n}$ is isomorphic to the expansion $\operatorname{Exp}\left(\tilde{\varepsilon}_{i, n}^{(1)}\right)$ of the 1-hull $\tilde{\varepsilon}_{i, n}^{(1)}$ of $\varepsilon_{i, n}$.

As stated in Theorem 1.2, if $n>3$ then $\Gamma_{i, n}$ is simply connected. Hence $\tilde{\varepsilon}_{i, n}=\varepsilon_{i, n}$ when $n>3$. On the other hand, $\tilde{\varepsilon}_{i, n}=\tilde{\varepsilon}_{i, n}^{(1)}$ when $n=3$ (recall that covers and 2-covers coincide in the rank 3 case). So, there is no need to study $\tilde{\varepsilon}_{i, n}$ here.

9.2. The universal 2-cover of $\Gamma_{1, n}$ and proof of claim (1) of Theorem 1.6

Proposition 9.2 The universal 2-cover of $\Gamma_{1, n}$ is the expansion of the free embedding of $P G(n-1,2)$ in $V\left(2^{n}-1,2\right)$.

Proof: Given a line $L$ of $P G(n-1,2)$, we have $L=\left\langle v_{1}, v_{2}\right\rangle$ for two non-zero vectors $v_{1}, v_{2}$ of $V(n, 2)$. Hence

$$
\varepsilon_{1, n}(L)=\left\{v_{1} \otimes v_{1}, v_{2} \otimes v_{2}, v_{1} \otimes v_{2}+v_{2} \otimes v_{1}\right\}
$$

The vectors $v_{1} \otimes v_{1}, v_{2} \otimes v_{2}$ and $v_{1} \otimes v_{2}+v_{2} \otimes v_{1}$ are independent. The conclusion follows from Lemma 9.1 and Proposition 2.8.

In view of Proposition 9.2 and since the codomain of $\varepsilon_{1, n}$ is $\left({ }^{n+1}{ }_{2}\right)$-dimensional, the universal 2 -cover of $\Gamma_{1, n}$ is a $2^{k}$-fold cover where $k=2^{n}-1-\left(\begin{array}{c}n+1 \\ 2\end{array}\right)$. In particular, the universal cover of $\Gamma_{1,3}$ is a double cover, as claimed in (1) of Theorem 1.6.

Remark 9.3 We can also describe the global kernel $K$ of $\varepsilon_{1, n}$. By the above, $\operatorname{dim}(K)=2^{k}$ with $k=2^{n}-1-\left({ }^{n+1}{ }_{2}\right)$. In particular, $\operatorname{dim}(K)=1$ when $n=3$. In fact, if $n=3$ then $K=\left\langle v_{P}\right\rangle$, where $v_{P}:=\sum_{p \in P} \iota(p), P$ stands for the point-set of $P G(2,2)$ and $\iota$ is the free embedding.

If $n>4$, then $K=\left\langle v_{S}\right\rangle_{S \in \mathcal{P}}$ where $\mathcal{P}$ is the collection of planes of $P G(n-1,2)$ and $v_{S}:=\sum_{p \in S} \iota(p)$. This follows by combining Corollary 2.6 with (4) of Section 2.4 when $n=4$ and with Corollary 2.7 when $n>4$.

\subsection{Proof of claim (2) of Theorem 1.6}

In view of Lemma 9.1, describing the universal 2-cover of $\Gamma_{2, n}$ amounts to compute the dimension of the 1-local kernel $\widetilde{K}^{(1)}$ of $\varepsilon_{2, n}$, but this computation is not so easy as in the case of $\varepsilon_{1, n}$. We will accomplish it only when $n=3$. We firstly describe $K_{L}$ for $L$ a line of $P G(n-1,4)$.

Lemma 9.4 The subspace $V_{L}=\left\langle\varepsilon_{2, n}(L)\right\rangle$ has dimension 4 and the five vectors of $\varepsilon_{2, n}(L)$ form an ovoid in $P G\left(V_{L}\right) \cong P G(3,2)$. 
Proof: Given two points $p_{\infty}:=\left\langle v_{1}\right\rangle$ and $p_{0}:=\left\langle v_{2}\right\rangle$ of $L$, the remaining three points of $L$ are the 1-dimensional linear subspaces of $V(n, 4)$ spanned by the vectors $v_{\lambda}=v_{2}+\lambda v_{1}$ for $\lambda \in G F(4) \backslash\{0\}$. With $p_{\lambda}:=\left\langle v_{\lambda}\right\rangle$ and $\omega \in G F(4) \backslash G F(2)$ as in the definition of $\varepsilon_{2, n}$, we have:

$$
\begin{aligned}
& \varepsilon_{2, n}\left(p_{\infty}\right)=v_{1} \otimes \bar{v}_{1}, \\
& \varepsilon_{2, n}\left(p_{0}\right)=v_{2} \otimes \bar{v}_{2}, \\
& \varepsilon_{2, n}\left(p_{1}\right)=v_{1} \otimes \bar{v}_{1}+v_{2} \otimes \bar{v}_{2}+v_{1} \otimes \bar{v}_{2}+v_{2} \otimes \bar{v}_{1}, \\
& \varepsilon_{2, n}\left(p_{\omega}\right)=v_{1} \otimes \bar{v}_{1}+v_{2} \otimes \bar{v}_{2}+v_{2} \otimes \bar{v}_{1}+\omega\left(v_{1} \otimes \bar{v}_{2}+v_{2} \otimes \bar{v}_{1}\right), \\
& \varepsilon_{2, n}\left(p_{1+\omega}\right)=\varepsilon_{2, n}\left(p_{\infty}\right)+\varepsilon_{2, n}\left(p_{0}\right)+\varepsilon_{2, n}\left(p_{1}\right)+\varepsilon_{2, n}\left(p_{\omega}\right) .
\end{aligned}
$$

Every 4-subset of the above quintuple of vectors is independent over $G F(2)$. The conclusion follows.

Corollary 9.5 $\operatorname{dim}\left(K_{L}\right)=1$.

(Trivial, by Lemma 9.4.) We will give a more explicit description of $K_{L}$ in a few lines, but we firstly state some notation. Denoted the point-set of $P G(n-1,4)$ by $P$, for every point $p \in P$ we put $v_{p}:=\iota(p)$ where, according to the notation of Section $2.4, \iota$ denotes the free embedding of $P G(n-1,4)$ in $V\left(\left(4^{n}-1\right) / 3,2\right)$. Also, we put $v_{X}:=\sum_{p \in X} v_{p}$ for $X \subseteq P$.

Lemma 9.6 $K_{L}=\left\langle v_{L}\right\rangle$.

Proof: As $\operatorname{dim}\left(K_{L}\right)=1$, we have $K_{L}=\left\langle v_{X}\right\rangle$ for a suitable nonempty subset $X$ of $L$. Furthermore, as $\varepsilon_{2, n}(L)$ is an ovoid of $P G\left(V_{L}\right)$, the subspaces $\left\langle v_{p}, v_{X}\right\rangle(p \in L)$ are 2dimensional, pairwise distinct and no four of them are contained in a common 4-dimensional subspace. The unique choice of $X$ that fulfils the above requirements is $X=L$.

Lemma 9.7 Let $n=3$. Then $\operatorname{dim}\left(\widetilde{K}^{(1)}\right)=10$.

Proof: Pick a conic $C$ and a dual conic $C^{*}$ of $P G(2,4)$, in such a way that all lines of $C^{*}$ are exterior to $C$. Let $\mathcal{T}$ be the bundle of lines of $P G(2,4)$ tangent to $C$ and put $\mathcal{B}:=\mathcal{T} \cup C^{*}$. We claim that the set $B:=\left\{v_{L}\right\}_{L \in \mathcal{B}}$ is a basis of $\widetilde{K}^{(1)}$.

We firstly prove that $B$ spans $\widetilde{K}^{(1)}$. Let $n$ be the nucleus of $C$ and $N$ the nuclear line of $C^{*}$. So, $C^{*} \cup\{N\}$ is the set of lines exterior to the hyperoval $C \cup\{n\}$. Every point of $N$ belongs to exactly one line of $C^{*}$ and, for every line $L \in C^{*}$, every point of $L$ different from $L \cap N$ belongs to exactly one line of $C^{*}$ different from $L$. Therefore,

$$
v_{N}=\sum_{L \in C^{*}} v_{L}
$$

Given a secant line $S$ of $C$, let $\left\{p_{1}, p_{2}\right\}=C \cap S$ and, for $i=1,2$, let $T_{i}$ be the line through $n$ and $p_{i}$. We can pick three lines $L_{1}, L_{2}, L_{3} \in C^{*} \cup\{N\}$ in such a way that none of the 
points $L_{1} \cap L_{2}, L_{2} \cap L_{3}$ and $L_{3} \cap L_{1}$ belongs to $S \cup T_{1} \cup T_{2}$. Then $\left\{S, T_{1}, T_{2}, L_{1}, L_{2}, L_{3}\right\}$ is a dual hyperoval. By the same argument used to prove (1) one can see that

$$
v_{S}=v_{T_{1}}+v_{T_{2}}+v_{L_{1}}+v_{L_{2}}+v_{L_{3}}
$$

By (1) and (2), $\langle B\rangle=\widetilde{K}^{(1)}$. It remains to prove that $B$ is independent.

Suppose that $\sum_{L \in \mathcal{X}} v_{L}=0$ for $\mathcal{X} \subseteq \mathcal{B}$. As every line of $\mathcal{T}$ contains one point that does not belong to any other line of $\mathcal{B}$, we have $\mathcal{X} \subseteq C^{*}$. However, every line of $C^{*}$ contains one point that does not belong to any other line of $C^{*}$. Hence $\mathcal{X}=\emptyset$. That is, $B$ is independent.

We are now ready to finish the proof of claim (2) of Theorem 1.6. By the previous lemma, $\operatorname{dim}\left(\widetilde{V}^{(1)}\right)=11$ when $n=3$. Since the codomain of $\varepsilon_{2,3}$ is 9-dimensional, $\operatorname{Exp}\left(\tilde{\varepsilon}_{2,3}^{(1)}\right)$ is a 4-fold cover of $\Gamma_{2,3}$.

Remark 9.8 Given a Fano subplane $S$ of $P G(2,4)$, the embedding induced by $\varepsilon_{2,3}$ on $S$ is isomorphic to $\varepsilon_{1,3}$. Hence $\Gamma_{2,3}$ contains 120 copies of $\Gamma_{1,3}$, as many as the Fano subplanes of $P G(2,4)$. By Remark 9.3, the global kernel $K$ of $\varepsilon_{2,3}$ contains $v_{S}$ for every Fano subplane $S$ of $P G(2,4)$. Hence $K \geq\left\langle v_{S}\right\rangle_{S \in \mathcal{P}}$, where $\mathcal{P}$ stands for the collection of Fano subplanes of $P G(2,4)$. In fact

$$
(*) K=\left\langle v_{S}\right\rangle_{S \in \mathcal{P}}
$$

but I am not going to prove this equality here. By combining Corollary 2.6 with Corollary 2.7 (or (4) of Section 2.4, when $n=4$ ) we obtain that $(*)$ also holds for any $n$. (Needless to say, in the general case $\mathcal{P}$ is the collection of Fano planes of $P G(n-1,4)$.)

\subsection{The universal 2-cover of $\Gamma_{2,4}$}

Lemma 9.9 If $n=4$, then $\operatorname{dim}\left(\widetilde{K}^{(1)}\right)=61$.

Proof: Given two points $p_{1}, p_{2}$ of $P G(3,4)$, let $L_{0}$ be the line joining them and, for $i=1,2$, pick six planes $S_{i, 0}, S_{i, 1}, \ldots, S_{i, 5}$ on $p_{i}$ in such a way that they form a dual hyperoval in the star of $p_{i}$ and $L_{0}$ is not contained in any of them. For $i, j=1,2, \ldots, 5$, put $L_{i, j}:=S_{1, i} \cap S_{2, j}$. Denoted by $\mathcal{L}_{1}$ the set of lines of $P G(3,4)$ incident to $p_{1}$ and by $\mathcal{L}_{2, i}$ the set of lines of $S_{2, i}$ on $p_{2}$, we set $\mathcal{L}_{2}:=\cup_{i=0}^{5} \mathcal{L}_{2, i}, \mathcal{L}_{3}:=\cup\left\{L_{i, j}\right\}_{i, j=1}^{5}, \mathcal{B}:=\mathcal{L}_{1} \cup \mathcal{L}_{2} \cup \mathcal{L}_{3}$ and $B:=\left\{v_{L}\right\}_{L \in \mathcal{B}}$.

Let $\overline{\mathcal{B}}$ be the set of lines $L$ with $v_{L} \in\langle B\rangle$. For $k=1,2, \ldots, 5, \mathcal{L}_{2, k} \cup\left\{L_{i, k}\right\}_{i=1}^{5}$ is a configuration of ten lines of $S_{2, k}$ as considered in the proof of Lemma 9.7. Hence $\overline{\mathcal{B}}$ contains all lines of $S_{2, k}$. Let $L$ be a line of $P G(3,4)$ skew with $L_{0}$, and $S$ the plane spanned by $L$ and $a$. By the above, the five lines $S \cap S_{i, 2}(i=1,2, \ldots, 5)$ belong to $\overline{\mathcal{B}}$. Furthermore, those five lines together with the five lines of $S$ through $p_{1}$ form in $S$ a configuration as in the proof of Lemma 9.7. Hence $L \in \overline{\mathcal{B}}$. Thus, $\overline{\mathcal{B}}$ contains all lines skew with $L_{0}$. It is now clear that $\overline{\mathcal{B}}$ contains all lines of $P G(3,4)$, namely $B$ spans $\widetilde{K}^{(1)}$. 
Suppose that $\mathcal{X} \subseteq \mathcal{B}$ is such that $\sum_{L \in \mathcal{X}} v_{L}=0$. The line $L_{0}$ has three points that do not belong to any other line of $\mathcal{B}$ and every line of $\mathcal{L}_{1} \backslash\left\{L_{0}\right\}$ contains one point that does not belong to any other line of $\mathcal{L}_{1}$. It follows that $\mathcal{X} \cap \mathcal{L}_{1}=\emptyset$. Similarly, every line of $\mathcal{L}_{2}$ contains one point that does not belong to any other line of $\mathcal{L}_{2} \cup \mathcal{L}_{3}$. Hence $\mathcal{X} \subseteq \mathcal{L}_{3}$. Finally, every line of $\mathcal{L}_{3}$ contains a point that does not belong to any other line of $\mathcal{L}_{3}$. Consequently, $\mathcal{X}=\emptyset$. Namely, $\mathcal{B}$ is independent. As $|B|=61$, we have $\operatorname{dim}\left(\widetilde{K}^{(1)}\right) \leq 61$.

Proposition 9.10 The universal 2-cover of $\Gamma_{2,4}$ is a $2^{8}$-fold cover.

Proof: By Lemma 9.9, $\operatorname{Exp}\left(\tilde{\varepsilon}_{2,4}^{(1)}\right)$, has exactly $2^{24}$ points. On the other hand, $\Gamma_{2,4}$ has $2^{16}$ points. The conclusion follows.

\section{Proof of claim (3) of Theorem 1.6 and end of the proof of Theorem 1.4}

In this section $\Gamma_{n}:=\operatorname{Far}_{\Delta}(F)$ for $\Delta=D_{n}(2)$ and $F$ a $\{+,-\}$-flag of $\Delta$.

\subsection{Preliminaries}

It is known [15] that $\Gamma_{n}$ admits a 2-quotient $\bar{\Gamma}_{n}$ with exactly $2^{n-1}$ elements of each of the two types + and - , where every (+)-element is incident to all ( - )-elements. That quotient is obtained by factorizing $\Gamma_{n}$ over the center of the unipotent radical of the stabilizer of $F$ in $O_{2 n}^{+}(2)$. More geometrically, $\bar{\Gamma}_{n}=\Gamma_{n} / \Theta$ where $\Theta$ is the equivalence relation defined as follows: for $\left\{U^{+}, U^{-}\right\}=F$ and $S:=U^{+} \cup U^{-}$as in Section 6, two elements $X, Y$ of $\Gamma_{n}$ correspond in $\Theta$ if and only if $X^{\perp} \cap S=Y^{\perp} \cap S$.

The first step of our investigation is to prove that the 0 -shadow geometry of $\bar{\Gamma}_{n}$ is the expansion of a suitable $G F(2)$-embedding, but we must describe that embedding first. Given a linear hyperplane $H$ of $V=V(n, 2)$, the vectors of $V \backslash H$ and the cosets $v+X$ for $v \in V \backslash H$ and $X$ a linear subspace of $H$ form a copy of $A G(n-1,2)$. Thus, we have a $G F(2)$-embedding of $A G(n-1,2)$ in $V(n, 2)$, which we call the natural $G F(2)$-embedding of $A G(n-1,2)$. We denote it by $v_{n}$.

Lemma 10.1 The 0-shadow geometry $\operatorname{Sh}_{0}\left(\bar{\Gamma}_{n}\right)$ of $\bar{\Gamma}_{n}$ is isomorphic to the dual of the expansion $\operatorname{Exp}\left(v_{n}\right)$ of the natural $G F(2)$-embedding $v_{n}$ of $A G(n-1,2)$.

Proof: We firstly revisit the construction of the geometry $\bar{\Gamma}_{n}$, describing it as the gluing of two copies of $A G(n-1,2)$, as in [15].

Recall that, for $\xi \in\{+,-\}, \operatorname{Res}_{\Delta}\left(U^{\xi}\right)$ can be regarded as a projective geometry $\mathcal{P}^{\xi} \cong$ $P G(n-1,2)$ with $U^{\xi}$ as the set of points. The set $S_{0}=U^{+} \cap U^{-}$is a hyperplane of $\mathcal{P}^{+}$. Thus, the elements of $\mathcal{P}^{\xi}$ that are not contained in $S_{0}$ form an affine geometry $\mathcal{A}^{\xi} \cong A G(n-1,2)$. The projective geometries $\mathcal{P}^{+}$and $\mathcal{P}^{-}$induce on $S_{0}$ the same geometry $\mathcal{P}_{0}\left(=\operatorname{Res}_{\Delta}(F)\right)$, which can be taken as the geometry at infinity of both $\mathcal{A}^{+}$and $\mathcal{A}^{-}$. Accordingly, given an element $X$ of $\mathcal{A}^{\xi}$ of dimension $\operatorname{dim}(X)>0$ and denoted by $\infty(X)$ its space at infinity (or its point at infinity, if $\operatorname{dim}(X)=1$ ), we have $\infty(X)=X \cap S_{0}$. (Note that, when doing so, we are 
regarding the points of $S_{0}$ as hyperplanes of $\mathcal{P}_{0}$.) It is now clear that, for $i \leq n-3$, the classes of $\Theta$ on the set of $i$-elements of $\Gamma_{n}$ bijectively correspond to the pairs $\left\{X^{+}, X^{-}\right\}$where $X^{+}$ and $X^{-}$are $(n-2-i)$-dimensional subspaces of $\mathcal{A}^{+}$and $\mathcal{A}^{-}$with $\infty\left(X^{+}\right)=\infty\left(X^{-}\right)$. For $\xi \in\{+,-\}$, the classes of $\Theta$ on the set of $\xi$-elements of $\Gamma_{n}$ bijectively correspond to the points of $\mathcal{A}^{\eta}$, where $\eta=\xi$ if $n$ is odd and $\{\xi, \eta\}=\{+,-\}$ if otherwise. Two pairs $\left\{X^{+}, X^{-}\right\}$and $\left\{Y^{+}, Y^{-}\right\}$as above correspond to incident elements of $\bar{\Gamma}_{n}=\Gamma_{n} / \Theta$ if and only if $X^{\xi}$ and $Y^{\xi}$ are incident in $\mathcal{A}^{\xi}$ for $\xi \in\{+,-\}$. For $\xi \in\{+,-\}$ a point $x \in \mathcal{A}^{\xi}$ and a pair $\left\{X^{+}, X^{-}\right\}$correspond to incident elements of $\bar{\Gamma}_{n}$ if and only if $x \in X^{\xi}$. As all (+)-elements of $\bar{\Gamma}_{n}$ are incident to all (-)-elements, every pair $\left\{x^{+}, x^{-}\right\}$with $x^{\xi}$ a point of $\mathcal{A}^{\xi}$ corresponds to a $\{+,-\}$-flag of $\bar{\Gamma}_{n}$.

We shall now rephrase the above in a slightly different way. The sets $U^{+}$and $U^{-}$are equipped with the right structures (namely, $\mathcal{P}^{+}$and $\mathcal{P}^{-}$) and have the right intersection for we may regard them as hyperplanes of some projective geometry $\mathcal{P}_{1} \cong P G(n, 2)$. Thus, $\mathcal{P}^{+}$ and $\mathcal{P}^{-}$are the projective geometries induced by $\mathcal{P}_{1}$ on $U^{+}$and $U^{-}$, the set $S_{0}=U^{+} \cap U^{-}$ is an $(n-2)$-dimensional subspace of $\mathcal{P}_{1}$ and $\mathcal{P}_{0}$ is the geometry induced by $\mathcal{P}_{1}$ on it. We keep for $\mathcal{A}^{+}$and $\mathcal{A}^{-}$the meaning stated above, namely $\mathcal{A}^{\xi}$ is the affine geometry obtained by removing $S_{0}$ from $\mathcal{P}^{\xi}$. As above, $\mathcal{P}_{0}$ is taken as the geometry at infinity of both $\mathcal{A}^{+}$ and $\mathcal{A}^{-}$.

Denoted by $U$ the hyperplane of $\mathcal{P}_{1}$ containing $S_{0}$ but different from $U^{+}$and $U^{-}$, let $\mathcal{P}$ be the projective geometry induced by $\mathcal{P}_{1}$ on $U, \mathcal{A} \cong A G(n-1,2)$ the complement of $S_{0}$ in $\mathcal{P}$ and $\mathcal{A}_{1} \cong A G(n, 2)$ the complement of $U$ in $\mathcal{P}_{1}$. For $j=1,2, \ldots, n-2$, given a pair $\left\{X^{+}, X^{-}\right\}$of $j$-dimensional subspaces of $\mathcal{A}^{+}$and $\mathcal{A}^{-}$with $\infty\left(X^{+}\right)=\infty\left(X^{-}\right)$, the set $X:=X^{+} \cup X^{-}$is a $(j+1)$-dimensional subspace of $\mathcal{A}_{1}$ with $S_{0} \nsubseteq \infty(X)$. Accordingly, $\infty(X)$ can be regarded as a $j$-dimensional subspace of $\mathcal{A}$. Thus, the elements of $\bar{\Gamma}_{n}$ of type $i=0,1, \ldots, n-3$ bijectively correspond to the $(n-i-1)$-dimensional subspaces of $\mathcal{A}_{1}$ with $(n-i-2)$-dimensional subspaces of $\mathcal{A}$ as their spaces at infinity. The set $S \backslash S_{0}=\left(U^{+} \cup U^{-}\right) \backslash S_{0}$ is the point-set of $\mathcal{A}_{1}$. So, the points of $\mathcal{A}_{1}$ bijectively correspond to the elements of $\bar{\Gamma}_{n}$ of type + and - . The lines of $\mathcal{A}_{1}$ with their point at infinity in $\mathcal{A}$ are precisely those that meet both $\mathcal{A}^{+}$and $\mathcal{A}^{-}$. So, those lines bijectively correspond to the $\{+,-\}$-flags of $\bar{\Gamma}_{n}$.

Thus, we have produced a copy of the 0 -shadow geometry of $\bar{\Gamma}_{n}$ inside $\mathcal{A}_{1}$. The conclusion is now obvious.

Remark 10.2 One might object that $\operatorname{Exp}\left(v_{n}\right)$ is isomorphic to the dual of $\operatorname{Sh}_{0}\left(\bar{\Gamma}_{n}\right)$ rather than to $\operatorname{Sh}_{0}\left(\bar{\Gamma}_{n}\right)$ itself, but the distinction between a geometry and its dual is not crucial here. We prefer not to insist on it.

As $\bar{\Gamma}_{n}$ is a 2-quotient of $\Gamma_{n}$, these two geometries have the same universal cover and the same universal 2-cover. Thus, by combining Lemma 10.1 with Proposition 2.5 and Proposition 2.2 we obtain the following:

Lemma 10.3 The expansion of the hull of $v_{n}$ is the 0-shadow geometry of the universal cover of $\Gamma_{n}$. The expansion of the 1-hull of $v_{n}$ is the 0-shadow geometry of the universal 2-cover of $\Gamma_{n}$. 


\subsection{The universal 2-cover of $\Gamma_{n}$ and proof of claim (3) of Theorem 1.6}

By Lemma 10.3 and Corollary 2.9 we immediately obtain the following:

Proposition 10.4 The 0-shadow geometry of the universal 2-cover of $\Gamma_{n}$ is the expansion $\operatorname{Exp}(\iota)$ of the free embedding เ of $A G(n-1,2)$ in $V\left(2^{n-1}, 2\right)$.

$\operatorname{Exp}(\iota)$ has $2^{2^{n-1}}$ points and the covering projection of $\operatorname{Exp}(\iota)$ onto $\Gamma_{n}$ maps them onto the elements of $\Gamma_{n}$ of type + and - . On the other hand, $\Gamma_{n}$ has $2^{1+\left(\begin{array}{c}n \\ 2\end{array}\right)}$ elements of type + and - . Hence the universal 2-cover of $\Gamma_{n}$ is a $2^{k}$-fold cover, with $k=2^{n-1}-\left(\begin{array}{c}n \\ 2\end{array}\right)-1$. In particular, the universal 2-cover of $\Gamma_{4}$ is a double cover.

Proof of Theorem 1.6(3): All residues of $\Gamma_{4}$ of rank 3 are simply connected (see Example 1.2 for those of type $\{+, 1,-\})$. Therefore, the universal cover and the universal 2 -cover of $\Gamma_{4}$ coincide. As noticed above, the latter is a double cover. Claim (3) of Theorem 1.6 is proved.

\subsection{Revisiting $\mathrm{Sh}_{0}\left(\Gamma_{n}\right)$ as an expansion}

Let $\bar{D}_{n}$ be the deck group of the projection from $\operatorname{Exp}(\iota)$ onto $\operatorname{Exp}\left(v_{n}\right)$. The 1-local kernel of $v_{n}$ is trivial (Corollary 2.9). Hence $\bar{D}_{n}$ is isomorphic to the global kernel $K$ of $v_{n}$ (Proposition 2.5). In fact, $\bar{D}_{n}$ is the same thing as $K$, but viewed as acting by addition on $\widehat{V}=V\left(2^{n-1}, 2\right)$, as a group of translations. Accordingly, all subgroups of $\bar{D}_{n}$ can be regarded as subspaces of $\widehat{V}$. In the sequel, we will freely switch from one to the other of these two points of view.

Every subgroup $D$ of $\bar{D}_{n}$ defines a 2-quotient $\operatorname{Exp}(\iota) / D$ of $\operatorname{Exp}(\iota)$. Denoted by $P$ the point-set of $A G(n-1,2)$, no two vectors of $\iota(P)$ belong to the same orbit of $D$. Indeed, if otherwise, the projection $\varphi: \operatorname{Exp}(\iota) \rightarrow \operatorname{Exp}(\iota) / D$ would send two distinct collinear points of $\operatorname{Exp}(\iota)$ onto the same point of $\operatorname{Exp}(\iota) / D$, contrary to the fact that $\varphi$ is a 2-covering. Therefore, $\iota$ induces a $G F(2)$-embedding $\iota / D$ of $A G\left(2^{n-1}, 2\right)$ in the quotient space $\widehat{V} / D$. Clearly, $\operatorname{Exp}(\iota) / D=\operatorname{Exp}(\iota / D)$. In particular, if $D_{n} \leq \bar{D}_{n}$ is the deck group of the projection of $\operatorname{Exp}(\iota)$ onto $\operatorname{Sh}_{0}\left(\Gamma_{n}\right)$, then

$$
\operatorname{Sh}_{0}\left(\Gamma_{n}\right) \cong \operatorname{Exp}\left(\varepsilon_{n}\right)
$$

where $\varepsilon_{n}:=\iota / D_{n}$. As $\operatorname{Exp}(\iota)$ is a $2^{2^{n-1}-\left(\begin{array}{l}n \\ 2\end{array}\right)-1}$-fold cover of $\operatorname{Sh}_{0}\left(\Gamma_{n}\right)$, we have

$$
\left|D_{n}\right|=2^{2^{n-1}-\left(\begin{array}{c}
n \\
2
\end{array}\right)-1} .
$$

Hence the codomain of $\varepsilon_{n}$ is $\left(\left(\begin{array}{c}n \\ 2\end{array}\right)+1\right)$-dimensional. Clearly, $D_{n}$ is the global kernel of $\varepsilon_{n}$.

Remark 10.5 The geometry $\Gamma_{n}$ is also an expansion, but in the more general sense of [16]: it is the expansion of the natural embedding of the line-grassmannian of $A G(n-1,2)$ in the 2nd-exterior power of $V(n, 2)$ (see [16, Lemma 8.4]). However, as line-grassmannians are not matroids, the handy machinery set up in Section 2.4 does not work for expansions like this. That is the reason why we have switched from $\Gamma_{n}$ to $\operatorname{Sh}_{0}\left(\Gamma_{n}\right)$. 


\subsection{End of the proof of Theorem 1.4}

We keep the notation of Section 10.3.

Lemma 10.6 We have $D_{4}=\left\langle v_{P}\right\rangle$, where $v_{P}:=\sum_{p \in P} \iota(p)$ and $P$ stands for the point-set of $A G(3,2)$.

Proof: By (2) of Section 10.3, $\operatorname{dim}\left(D_{4}\right)=1$. Hence $D_{4}=\left\langle v_{x}\right\rangle$, where $v_{X}=\sum_{p \in X} \iota(p)$ for a suitable nonempty subset $X$ of $P$. On the other hand, as $\operatorname{Exp}(\iota) / D_{4} \cong \operatorname{Sh}_{0}\left(\Gamma_{4}\right)$ is flag-transitive, the normalizer $N$ of $D_{4}$ in $\operatorname{Aut}(\operatorname{Exp}(\iota))$ is flag-transitive on $\operatorname{Exp}(\iota)$ (see [13, Theorem 12.59]). In particular, $N$ induces on $\iota(P)$ a transitive permutation group $N_{P}$. However, as $N$ normalizes $D_{4}$ and $D_{4}$ is spanned by $v_{X}, N_{P}$ is forced to stabilize $X$. Hence $X=P$.

Lemma 10.7 We have $D_{5}=\left\langle v_{S}\right\rangle_{S \in \mathcal{P}}$, where $v_{S}:=\sum_{p \in S} \iota(p)$ and $\mathcal{P}$ is the collection of planes of $A G(4,2)$.

Proof: By Lemma 10.6, $K_{S}=\left\langle v_{S}\right\rangle$ for every $S \in \mathcal{P}$. Hence $D_{5}$, which is the global kernel of $\varepsilon_{5}$, contains $W:=\left\langle v_{S}\right\rangle_{S \in \mathcal{P}}$. However, $\operatorname{dim}\left(D_{5}\right)=5$ by (2) of Section 10.3. So, we only need to prove that $\operatorname{dim}(W) \geq 5$.

Given a minimal spanning set $\left\{p_{i}^{\infty}\right\}_{i=1}^{4}$ of the space at infinity of $A G(4,2)$ and a point $p$ of $A G(4,2)$, for $k=1,2,3,4$ let $S_{k}$ be the 3-space of $A G(4,2)$ containing $p$ and with $\left\langle p_{i}^{\infty}\right\rangle_{i \neq k}$ as the plane at infinity. Let $S_{5}$ be the 3 -space of $A G(4,2)$ parallel to $S_{1}$. By a routine computation one can check that the vectors $v_{S_{1}}, v_{S_{2}}, v_{S_{3}}, v_{S_{4}}$ and $v_{S_{5}}$ are linearly independent. Hence $\operatorname{dim}(W) \geq 5$.

We can now finish the proof of Theorem 1.4. By Lemma 10.7, the local kernel and the global kernel of $\varepsilon_{5}$ coincide. Hence $\operatorname{Exp}\left(\varepsilon_{5}\right)$ is simply connected, by Corollary 2.6. As $\mathrm{Sh}_{0}\left(\Gamma_{5}\right) \cong \operatorname{Exp}\left(\varepsilon_{5}\right)\left(\right.$ Section 10.3, (1)), $\mathrm{Sh}_{0}\left(\Gamma_{5}\right)$ is simply connected, too. Therefore $\Gamma_{5}$ is simply connected, by the first part of Proposition 2.2.

Remark 10.8 The equality $D_{n}=\left\langle v_{S}\right\rangle_{S \in \mathcal{P}}$, with $\mathcal{P}$ the collection of planes of $A G(n-1,2)$, also holds when $n>5$, as it follows from Lemma 10.7 and Corollaries 2.6 and 2.7. We refer to Baumeister, Meixner and Pasini [2] for more information.

\section{References}

1. B. Baumeister and G. Stroth, "The non-canonical gluings of two affine spaces," in Groups and Geometries, L. Di Martino et al. (Eds.), Birkhäuser, 1998, pp. 9-28.

2. B. Baumeister, T. Meixner, and A. Pasini, "GF(2)-expansions," Geom. Dedicata 67 (1997), 163-180.

3. B. Baumeister, S. Shpectorov, and G. Stroth, "Flag-transitive affine dual polar spaces," preprint, July 1997.

4. R.J. Blok and A.E. Brouwer, "The geometry far from a residue," in Groups and Geometries, L. Di Martino et al. (Eds.), Birkhäuser, 1998, pp. 29-38.

5. A.E. Brouwer, A.M. Cohen, and A. Neumaier, Distance Regular Graphs, Springer, Berlin, 1989.

6. F. Buekenhout, Handbook of Incidence Geometry, Elsevier, Amsterdam, 1995.

7. A. Cohen and E. Shult, "Affine polar spaces," Geom. Dedicata 35 (1990), 43-76. 
8. H. Cuypers and A. Pasini, "Locally polar geometries with affine planes," European J. Combin. 13 (1992), 39-57.

9. C. Huybrechts and A. Pasini, "Flag-transitive extensions of dual projective spaces," Bull. Soc. Math. Belgique 5 (1998), 341-352.

10. A. Munemasa and S.V. Shpectorov, "A local characterization of the graphs of alternating forms," in Finite Geometry and Combinatorics, F. De Clerck et al. (Eds.), Cambridge Univ. Press, 1993, pp. 289-302.

11. A. Munemasa, D.V. Pasechnik, and S.V. Shpectorov, "A local characterization of the graphs of alternating forms and the graphs of the quadratic forms over GF(2)," in Finite Geometry and Combinatorics, F. De Clerck et al. (Eds.), Cambridge Univ. Press, 1993, pp. 303-317.

12. A. Pasini, "On locally polar geometries whose planes are affine," Geom. Dedicata 34 (1990), 35-56.

13. A. Pasini, Diagram Geometry, Oxford Univ. Press, 1994.

14. A. Pasini, "Shadow geometries and simple connectedness," European J. Combin. 15 (1994), 17-34.

15. A. Pasini, "Gluing two affine spaces," Bull. Belgian Math. Soc. 3 (1996), 25-40.

16. A. Pasini, "Embeddings and expansions," Bull. Belgian Math. Soc. to appear.

17. S. Rinauro, "On some extensions of generalized quadrangles of grid type," J. Geometry 38 (1990), 158-164.

18. J. Tits, Buildings of Spherical Type and Finite BN-pairs, Springer Lect. Notes 1974, vol. 386.

19. L. Van Nypelseer, "Rank $n$ geometries with affine hyperplanes and dual affine point residues," European $J$. Combin. 12 (1991), 561-566. 